\title{
Orientational prewetting of planar solid substrates by a model liquid crystal
}

Cite as: J. Chem. Phys. 135, 204702 (2011); https://doi.org/10.1063/1.3660377

Submitted: 16 August 2011 • Accepted: 21 October 2011 • Published Online: 22 November 2011

Manuel Greschek and Martin Schoen

\section{ARTICLES YOU MAY BE INTERESTED IN}

Topological defects around a spherical nanoparticle in nematic liquid crystal: Coarse-grained molecular dynamics simulations

The Journal of Chemical Physics 141, 114903 (2014); https://doi.org/10.1063/1.4894438

Simulation of Gay-Berne liquid crystal molecules confined to a plane

AIP Conference Proceedings 2220, 130016 (2020); https://doi.org/10.1063/5.0001132

Effects of flow on topological defects in a nematic liquid crystal near a colloid

The Journal of Chemical Physics 140, 054905 (2014); https://doi.org/10.1063/1.4862953

The Journal

SPECIAL TOPIC: Low-Dimensional

of Chemical Physics Materials for Quantum Information Science 


\title{
Orientational prewetting of planar solid substrates by a model liquid crystal
}

\author{
Manuel Greschek ${ }^{1, a)}$ and Martin Schoen ${ }^{1,2, b)}$ \\ ${ }^{1}$ Stranski-Laboratorium für Physikalische und Theoretische Chemie, Fakultät für Mathematik und \\ Naturwissenschaften, Technische Universität Berlin, Straße des 17. Juni 135, 10623 Berlin, Germany \\ ${ }^{2}$ Department of Chemical and Biomolecular Engineering, Engineering Building I, Box 7905, North Carolina \\ State University, 911 Partners Way, Raleigh, North Carolina 27695, USA
}

(Received 16 August 2011; accepted 21 October 2011; published online 22 November 2011)

\begin{abstract}
We present grand canonical ensemble Monte Carlo simulations of prewetting transitions in a model liquid crystal at structureless solid substrates. Molecules of the liquid crystal interact via anisometric Lennard-Jones potentials and can be anchored planar or homeotropically at the substrates. Fluidsubstrate attraction is modeled by a Yukawa potential of variable range. By monitoring the grandpotential density and the nematic order parameter as functions of the chemical potential $\mu$, several discontinuous prewetting, wetting, and isotropic-nematic phase transitions are observed. These transitions depend on both the range of the fluid-substrate attraction and the specific anchoring at the substrate. Our results show that at substrates characterized by degenerate anchoring prewetting occurs at lower $\mu$ compared with cases in which the anchoring is monostable. This indicates that prewetting transitions are driven by orientational entropy because degenerate anchoring allows for more orientationally distinct configurations of molecules compared with monostable anchoring. In addition, by analyzing local density and various local order parameters, a detailed picture of the structure of various phases emerges from our simulations. (C) 2011 American Institute of Physics. [doi:10.1063/1.3660377]
\end{abstract}

\section{INTRODUCTION}

If a liquid crystal is in contact with a solid substrate, the presence of that substrate may induce specific preferred orientations of the molecules in its vicinity even though in the bulk the liquid crystal would be isotropic under identical thermodynamic conditions. The preferred orientation of molecules in the immediate vicinity of the solid substrate is a consequence of "anchoring". ${ }^{1}$ Anchoring may be perceived as an energetic discrimination of a set of molecular orientations. According to the definitions given by Jerôme, ${ }^{1}$ anchoring conditions may be classified as degenerate if an infinite number of so-called "easy axes" exists, monostable if the molecules align with a single preferred spatial direction, or multistable if a finite number of orientations exists that are energetically discriminated by a substrate. Perhaps, the earliest study of how anchoring may affect properties of a liquid-crystal film has been carried out by Sheng within the framework of Landaude Gennes theory. ${ }^{2,3}$ Depending on the thermodynamic conditions, Sheng observed an isotropic-nematic phase transition occurring either near the solid substrate or far away from the substrate. ${ }^{3}$ Shortly after these early theoretical studies, it was shown experimentally by Miyano ${ }^{4}$ that liquid-crystal films adsorbed on solid substrates may exhibit birefringence indicating a significant degree of nematic order present in these films. Understanding and eventually controlling the impact of anchoring on wetting of solid surfaces is not only of academic interest but may also have important repercussions in the realm of optoelectronic devises, for example. ${ }^{5}$

\footnotetext{
${ }^{a)}$ Electronic mail: manuel.greschek@tu-berlin.de.

b)Electronic mail: martin.schoen@tu-berlin.de.
}

Therefore, it does not seem surprising that a host of experimental techniques exists to realize substrates with specific anchoring characteristics. According to Sonin, three major groups can be identified: mechanical work, chemical substance deposition, and external action. ${ }^{6}$ For instance, a substrate can be exposed to mechanical work by rubbing or polishing it, by exposing it to photolithographic relief, or by oblique evaporation of oxide films. Chemical substance deposition can be realized either through deposition of surfactants or polymeric films. The former causes homeotropic anchoring whereas planar anchoring is realized by the latter approach. Finally, external fields or flow can be used to manipulate the anchoring of molecules at substrate surfaces as examples of external action. Depending on details of how anchoring is effected, molecules that are located at a larger/smaller distance from the substrate surface may be aligned in a specific fashion. This, of course, requires a fluid-substrate interaction potential of variable range. A more detailed discussion and references to relevant literature can be found in Chapter 2 of Sonin's book. ${ }^{6}$

More recently, and as far as preparation of solid substrates with specific anchoring is concerned, the focus has shifted towards nanometer-size structures and heterogeneous substrates. An example is the work by Yi et al. who use polymer films nanoimprinted with checkerboard patterns of square wells to demonstrate that the geometry of these wells allows one to align liquid-crystal molecules in different ways. ${ }^{7}$ They confirm their experimental findings also by simple theoretical considerations.

Once the substrate has been endowed with specific anchoring characteristics the resulting structure of an adjacent liquid crystal has most frequently been studied by 
ellipsometry. For example, Hsiung et $a l .{ }^{8}$ have studied the substrate-induced orientational order in an otherwise isotropic liquid crystal and observe a weak nematic layer on a silane-decorated glass substrate. The transition from molecularly thin to thick films has been investigated by Valignat et al. for droplets of nonvolatile liquids using spatially resolved ellipsometry. ${ }^{9}$ Chen et al. studied the orientational wetting of a homologous series of cyanobiphenyl liquid crystals on glass that was coated with silane surfactant molecules. ${ }^{10}$ Lucht and Bahr used ellipsometry to investigate the wetting behavior at the free surface of the isotropic phase of binary mixtures of thermotropic liquid crystals. ${ }^{11}$ More recently, Bahr also reported a prewetting transition at the interface between a nematic liquid crystal and water in the presence of nonionic surfactant. ${ }^{12}$ In this work, it is also demonstrated that increasing surfactant concentration drives the prewetting transition towards a surface-critical point. Experimental evidence for the existence of an orientational prewetting transition is also provided through specific-heat measurements carried out by Liu and co-workers. ${ }^{13}$

On the theoretical side, there has also been some interest in wetting of solid substrates by liquid-crystalline materials. Quite some time ago, Poniewierski and Sluckin have studied wetting transitions. ${ }^{14}$ They employ a version of Maier-Saupe mean-field theory properly modified for homeotropically anchoring substrates. In their study, prewetting of the substrate by a nematic phase is observed. Later, these same authors used Landau-de Gennes' theory to demonstrate that close to the bulk isotropic-nematic phase transition the nematic film near the substrate may be perceived as a low-temperature Kosterlitz-Thouless phase. ${ }^{15}$ Landau-de Gennes theory has been rather popular in the field of wetting phenomena. ${ }^{16-18}$ Another interesting example is the work by Patrício et al. ${ }^{19}$ These authors studied wetting transitions of nematic liquid crystals on periodic, wedge-shaped substrates.

Using instead mean-field density functional theory Telo da Gama investigated prewetting of solid substrates by liquid crystals. ${ }^{20}$ If the fluid-substrate interaction decays exponentially with increasing distance from the substrate, the prewetting transition depends on how rapid this decay actually is. Later, de las Heras and co-workers used a version of Onsager's theory ${ }^{21}$ to study phase transitions in a fluid composed of hard spherocylinders confined to a slit-pore. ${ }^{22,23}$ In the limit of a large distance between the substrates interesting wetting phenomena involving different mesophases are observed. However, prewetting does not occur in this system.

In addition, computer simulations have frequently been employed to study orientational wetting of substrates by liquid crystals. Dijkstra et al. investigated fluids composed of hard spherocylinders interacting with hard substrates by Monte Carlo (MC) simulation. ${ }^{24}$ By means of density functional theory and MC simulation the same authors ${ }^{25}$ studied wetting phenomena within Zwanzig's ${ }^{26}$ simple hard-rod model. MC simulations have been performed by Allen ${ }^{27}$ to investigate the impact of anchoring scenarios at solid substrates on nematic liquid-crystalline phases. Again by means of MC simulations, Downton and Allen investigated the impact of various anchoring scenarios by grafting hard spherocylinders homeotropically onto hard substrates. ${ }^{28}$ By varying both the grafting density and the length of the grafted molecules, a number of novel anchoring regimes in the adjacent liquid-crystal phase could be observed. Using the Gay-Berne model for a system of soft disks Bellier-Castella et al. could demonstrate that the temperature of the nematiccolumnar transition depends on the substrate anchoring of the disks. ${ }^{29}$ The Gay-Berne model has also been employed by Müller et al. who observed that the formation of molecularly thick films adsorbed on solid substrates does not involve a prewetting transition under the conditions employed in their simulations. ${ }^{30}$ By using a fluid composed of hard Gaussian overlap particles interacting with planar substrates through a hard-needle potential, Barmes and Cleaver were able to realize both planar and homeotropic anchoring scenarios. ${ }^{31}$ In their model, the substrate interacts with a "needle" of variable length embedded inside a fluid molecule. If the length of the needle corresponds to the long axis of a molecule, planar alignment at the substrate results; if, on the other hand, the needle shrinks to a point, homeotropic alignment is observed. ${ }^{31}$ Cheung used MC simulations to demonstrate that structured substrates may affect the alignment of molecules. ${ }^{32}$ Here, even tilted alignment of molecules could be realized by fine-tuning the fluid-substrate interaction. Similarly, Zhang et al. could show that a substrate may control the alignment of molecules in (bulk-like) regions of an adjacent liquid-crystal phase. $^{33}$

In this paper, we will focus on the impact of various anchoring scenarios on the prewetting transition and how this transition depends on the range of the fluid-substrate interaction potential. We employ a simple model based upon an anisotropic Lennard-Jones potential introduced by Hess and $\mathrm{Su}^{34}$ and employed by us in previous studies. ${ }^{35-37}$ The remainder of this paper is organized as follows. In Sec. II, we introduce the model on which our study is based. We summarize the basic theoretical concepts in Sec. III. Results are presented in Sec. IV and put into perspective in the concluding Sec. V. Relevant general properties of the grand-potential density are summarized in the Appendix.

\section{MODEL SYSTEM}

We consider a fluid composed of $N$ molecules interacting with each other in a pairwise additive fashion such that the fluid-fluid (ff) configurational potential energy is given by

$$
U_{\mathrm{ff}}(\boldsymbol{R}, \widehat{\boldsymbol{U}})=\frac{1}{2} \sum_{i=1}^{N} \sum_{j \neq i}^{N} u_{\mathrm{ff}}\left(\boldsymbol{r}_{i j}, \widehat{\boldsymbol{u}}_{i}, \widehat{\boldsymbol{u}}_{j}\right),
$$

where $\boldsymbol{r}_{i j} \equiv \boldsymbol{r}_{i}-\boldsymbol{r}_{j}$ is the distance vector between the centers of mass of particles $i$ and $j$ located at $\boldsymbol{r}_{i}$ and $\boldsymbol{r}_{j}$, respectively. In Eq. (2.1), $\boldsymbol{R} \equiv\left\{\boldsymbol{r}_{1}, \boldsymbol{r}_{2}, \ldots, \boldsymbol{r}_{N}\right\}$ and $\widehat{\boldsymbol{U}} \equiv\left\{\widehat{\boldsymbol{u}}_{1}, \widehat{\boldsymbol{u}}_{2}, \ldots, \widehat{\boldsymbol{u}}_{N}\right\}$ are shorthand notations for the respective sets of center-ofmass coordinates and unit vectors specifying the orientations of the liquid-crystalline molecules. Following earlier work, ${ }^{34-39}$ we adopt the intermolecular interaction potential 


$$
\begin{aligned}
u_{\mathrm{ff}}\left(\boldsymbol{r}_{i j}, \widehat{\boldsymbol{u}}_{i}, \widehat{\boldsymbol{u}}_{j}\right)= & 4 \epsilon\left[\left(\frac{\sigma}{r_{i j}}\right)^{12}-\left(\frac{\sigma}{r_{i j}}\right)^{6}\right. \\
& \left.\times\left\{1+\Psi\left(\widehat{\boldsymbol{r}}_{i j}, \widehat{\boldsymbol{u}}_{i}, \widehat{\boldsymbol{u}}_{j}\right)\right\}\right],
\end{aligned}
$$

where $r=|\boldsymbol{r}|$ and $\widehat{\boldsymbol{r}}=\boldsymbol{r} / r$. Throughout this work, notation "..." indicates a unit vector. Hence, $u_{\mathrm{ff}}$ is a Lennard-Jones potential where the attractive contribution is modified to account for different relative orientations of a pair of molecules. In Eq. (2.2), $\sigma$ denotes the "diameter" of a spherical reference molecule and $\epsilon$ is the depth of the attractive well in that reference model. The anisotropy of the fluid-fluid interaction is accounted for by the function

$$
\begin{aligned}
\Psi\left(\widehat{\boldsymbol{r}}_{i j}, \widehat{\boldsymbol{u}}_{i}, \widehat{\boldsymbol{u}}_{j}\right)= & 5 \varepsilon_{1} P_{2}\left(\widehat{\boldsymbol{u}}_{i} \cdot \widehat{\boldsymbol{u}}_{j}\right) \\
& +5 \varepsilon_{2}\left[P_{2}\left(\widehat{\boldsymbol{u}}_{i} \cdot \widehat{\boldsymbol{r}}_{i j}\right)+P_{2}\left(\widehat{\boldsymbol{u}}_{j} \cdot \widehat{\boldsymbol{r}}_{i j}\right)\right],
\end{aligned}
$$

which one obtains from a summation of certain Wigner matrices selected to preserve the head-tail symmetry of the molecules $^{34}$ and to guarantee that

$$
\iint \mathrm{d} \widehat{\boldsymbol{u}}_{i} \mathrm{~d} \widehat{\boldsymbol{u}}_{j} \Psi\left(\widehat{\boldsymbol{r}}_{i j}, \widehat{\boldsymbol{u}}_{i}, \widehat{\boldsymbol{u}}_{j}\right)=0,
$$

which holds because in Eq. (2.3),

$$
P_{2}(x)=\frac{1}{2}\left(3 x^{2}-1\right),
$$

is the second Legendre polynomial. In other words, $\Psi$ remains unaltered if $\widehat{\boldsymbol{u}}_{i}$ and/or $\widehat{\boldsymbol{u}}_{j}$ are replaced by $-\widehat{\boldsymbol{u}}_{i}$ and/or $-\widehat{\boldsymbol{u}}_{j}$ (head-tail symmetry).

In Eq. (2.3), $\varepsilon_{1}$ and $\varepsilon_{2}$ are (dimensionless) anisotropy parameters determining the orientation dependence of intermolecular attractions. In this study, we deviate from our previous work ${ }^{35-37}$ and choose $\varepsilon_{1}=0.04$ and $\varepsilon_{2}=-0.04$. With

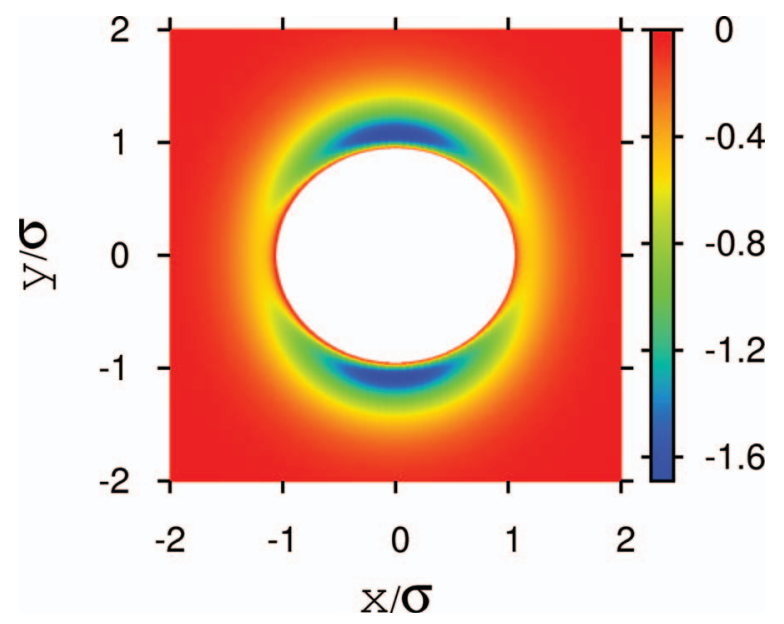

FIG. 1. Plots of $u\left(\boldsymbol{r}_{12}, \widehat{\boldsymbol{u}}_{1}, \widehat{\boldsymbol{u}}_{2}\right)$ for a pair of molecules located in the $x-y$ plane such that $\boldsymbol{r}_{12}=\left(x_{12}, y_{12}, 0\right)$ and $\widehat{\boldsymbol{u}}_{1} \cdot \widehat{\boldsymbol{u}}_{2}=1$. The white area at the center of the plot is defined by the condition $u_{\mathrm{ff}}\left(\boldsymbol{r}_{12}, \widehat{\boldsymbol{u}}_{1}, \widehat{\boldsymbol{u}}_{2}\right) \geq 0$ such that it approximately represents the shape of the molecule at the center of the coordinate system true to scale. In colored parts of the figure $u_{\mathrm{ff}}\left(\boldsymbol{r}_{12}, \widehat{\boldsymbol{u}}_{1}, \widehat{\boldsymbol{u}}_{2}\right)<0$ where the color indicates the local value $u_{\mathrm{ff}}$ in units of $\epsilon$ (see attached color bar). this choice, molecules are nearly spherical as the plot in Fig. 1 reveals. Taking the ratio of zeros of $u_{\mathrm{ff}}$ in Eq. (2.2) for side-side $\left(\widehat{\boldsymbol{u}}_{1} \cdot \widehat{\boldsymbol{u}}_{2}= \pm 1, \widehat{\boldsymbol{u}}_{1} \cdot \widehat{\boldsymbol{r}}=\widehat{\boldsymbol{u}}_{2} \cdot \widehat{\boldsymbol{r}}=0\right)$ and end-end configurations $\left(\widehat{\boldsymbol{u}}_{1} \cdot \widehat{\boldsymbol{u}}_{2}= \pm 1, \widehat{\boldsymbol{u}}_{1} \cdot \widehat{\boldsymbol{r}}=\widehat{\boldsymbol{u}}_{2} \cdot \widehat{\boldsymbol{r}}= \pm 1\right)$ as a definition of the aspect ratio $\kappa$ of a fluid molecule, we obtain $\kappa$ $\simeq 1.095$. The main purpose for selecting $\varepsilon_{2}=-0.04$ is that this reduces the overall fluid-fluid attraction compared with $\varepsilon_{2}$ $=-0.08$ used in our previous works. ${ }^{35-37}$ As a consequence, the density range of the bulk one-phase gas region is considerably wider for temperatures that are not too much below the (estimated) gas-(isotropic) liquid critical temperature. ${ }^{34}$

The fluid is confined between two planar, structureless solid substrates separated by a distance $s_{z 0}$ along the $z$-axis of a space-fixed Cartesian coordinate system. Hence, a fluidsubstrate (fs) contribution to the total configurational energy may be cast as

$$
U_{\mathrm{fs}}(\boldsymbol{R}, \widehat{\boldsymbol{U}})=\sum_{k=1}^{2} \sum_{i=1}^{N} u_{\mathrm{fs}}^{[k]}\left(\boldsymbol{r}_{i}, \widehat{\boldsymbol{u}}_{i}\right),
$$

where

$$
\begin{aligned}
u_{\mathrm{fs}}^{[k]}\left(z_{i}, \widehat{\boldsymbol{u}}_{i} ; \eta\right)= & \epsilon\left[a_{1}\left(\frac{\sigma}{z_{i} \pm s_{\mathrm{z} 0} / 2}\right)^{10}\right. \\
& \left.-a_{2} \frac{\exp \left(-\eta\left|z_{i} \pm s_{\mathrm{z} 0} / 2\right|\right)}{\left|z_{i} \pm s_{\mathrm{z} 0} / 2\right|} g^{[k]}\left(\widehat{\boldsymbol{u}}_{i}\right)\right]
\end{aligned}
$$

where $z_{i}=\boldsymbol{r}_{i} \cdot \widehat{\boldsymbol{e}}_{\mathrm{z}}$ and $\widehat{\boldsymbol{e}}_{\mathrm{z}}$ points in the direction of the $z$-axis of a space-fixed Cartesian coordinate system. We assume the lower substrate $(k=1)$ to be located at $z_{\mathrm{w}}=-s_{\mathrm{z} 0} / 2$ whereas the upper one is located at $z_{\mathrm{w}}=+s_{\mathrm{z} 0} / 2(k=2)$. Because the substrates are structureless, $u_{\mathrm{fs}}$ at fixed molecular orientation depends only on the center-of-mass distance of molecule $i$ from either substrate along the $z$-axis. Moreover, because we are interested in a physical situation in which a molecule might interact with a single substrate at most we fix $s_{\mathrm{z} 0}=50 \sigma$ throughout this work.

The orientation dependence of the fluid-substrate interaction is described by the so-called anchoring function $0 \leq g^{[k]}\left(\widehat{\boldsymbol{u}}_{i}\right) \leq 1$ which we specify in Table I. We consider combinations of anchoring functions that favor either planar or homeotropic alignment. Notice that for nonspecific (n) anchoring $(g=1)$, no orientation of a molecule with respect to the substrate plane is energetically favored. According to the classification scheme of Jerôme ${ }^{1}$, this results in degenerate anchoring. Nevertheless, molecules prefer an on-average homeotropic alignment. Notice that in our model, only the center of mass of a molecule interacts with the substrate. This is also the case in the model of Barmes and Cleaver if their interaction "needle" embedded inside the elongated molecules shrinks to a point (see Sec. I). ${ }^{31}$ In our model, the fluid-fluid potential favors side-side arrangements of a pair of molecules and stabilizes the homeotropic alignment at the substrate surfaces. In addition, because of $\kappa>1$ packing at the surface plane is slightly more efficient if the weakly nonspherical molecules are homeotropically rather than planar oriented. As far as planar anchoring is concerned, the anchoring scenario 
TABLE I. Combinations of anchoring functions $g^{[k]}(\widehat{\boldsymbol{u}})$ used in this study [see Eq. (2.7)]. Combinations of planar (p) and directional (d) as well as of nonspecific (n) and homeotropic (h) anchoring are considered. Unit vector $\widehat{\boldsymbol{e}}_{\alpha}$ points along the $\alpha$-direction of a space-fixed Cartesian coordinate system.

\begin{tabular}{lcc}
\hline \hline Acronym & $g^{[1]}(\widehat{\boldsymbol{u}})$ & $g^{[2]}(\widehat{\boldsymbol{u}})$ \\
\hline pd & $\left(\widehat{\boldsymbol{u}} \cdot \widehat{\boldsymbol{e}}_{\mathrm{X}}\right)^{2}+\left(\widehat{\boldsymbol{u}} \cdot \widehat{\boldsymbol{e}}_{\mathrm{y}}\right)^{2}$ & $\left(\widehat{\boldsymbol{u}} \cdot \widehat{\boldsymbol{e}}_{\mathrm{X}}\right)^{2}$ \\
$\mathrm{nh}$ & 1 & $\left(\widehat{\boldsymbol{u}} \cdot \widehat{\boldsymbol{e}}_{\mathrm{Z}}\right)^{2}$ \\
\hline \hline
\end{tabular}

referred to as directional (d) pins the molecules' longer axes to the $x$-direction whereas in the planar (p) scenario all orientations on a unit circle in the substrate plane is energetically equivalent. Again, according to Jerôme's classification scheme, the former causes monostable anchoring whereas in the latter case anchoring is degenerate.

In Eq. (2.7), we employ a Yukawa-like attractive contribution, where $\eta^{-1}$ may be interpreted as a "screening length" which determines the range of the fluid-substrate attraction. Notice that the Yukawa-type of attraction-usually describing the interaction between screened charges-has been chosen without any specific experimental system in mind. Rather this form of fluid-substrate attraction has been selected out of mere convenience because it permits us to tune the range of this attraction continuously by varying $\eta$. In this work, we consider $\eta \sigma=0.25,0.45$, and 1.0 such that with increasing $\eta$ the fluid-substrate attraction becomes increasingly shorter range. Dimensionless parameters,

$$
\begin{aligned}
& a_{1} \equiv-\frac{15}{4} \frac{\eta \sigma+1}{\eta \sigma-9}, \\
& a_{2} \equiv-\frac{75}{2} \frac{\exp (\eta \sigma)}{\eta \sigma-9},
\end{aligned}
$$

have been introduced to guarantee that

$$
\begin{gathered}
\left.\frac{\mathrm{d} u_{\mathrm{fs}}^{[k]}}{\mathrm{d} z}\right|_{z_{\min }}=0, \\
u_{\mathrm{fs}}^{[k]}\left(z_{\min }\right)=-\frac{15}{4} \epsilon,
\end{gathered}
$$

irrespective of $\eta \sigma$, where $z_{\min }= \pm s_{\mathrm{z} 0} / 2 \mp \sigma$ denotes the location of the minimum of $u_{\mathrm{fs}}$ relative to the upper and lower substrate surface, respectively. The depth of the attractive well at $z=z_{\min }$ in Eq. (2.2) has been chosen arbitrarily but large enough to guarantee complete wetting of the substrates as one approaches gas-(isotropic) liquid coexistence. We illustrate the various fluid-substrate potentials in Fig. 2. The plots show that with our choice of $a_{1}$ and $a_{2}$ only the range of the attractive part of the total fluid-substrate attraction is affected by the specific choice of $\eta \sigma$. From the inset in Fig. 2, it is also clear that molecules located near the midpoint $z=0$ are nearly unaffected by the presence of either wall because $u_{\mathrm{fs}}^{[1]}+u_{\mathrm{fs}}^{[2]} \approx 0$ irrespective of the value of $\eta \sigma$ considered in this study. In other words, portions of the liquid crystal in the vicinity of one wall are indeed not subject to a simultaneous interaction with the other one.

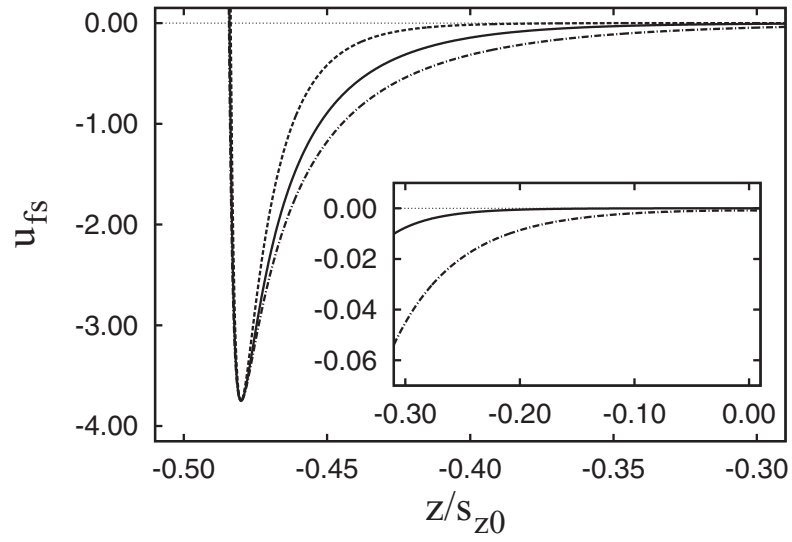

FIG. 2. Total fluid-substrate interaction potential $u_{\mathrm{fs}}^{[1]}+u_{\mathrm{fs}}^{[2]}$ as a function of position $z$ in the lower half of the system and for $\eta=0.25(-\cdot-\cdot), \eta=0.45$ (一), and $\eta=1.00$ (----) [see Eq. (2.7)]. Inset shows an enhancement close to the midpoint of the system. Data for $\eta=1.00$ are omitted because they are indistinguishable from $u_{\mathrm{fs}}^{[1]}+u_{\mathrm{fs}}^{[2]}=0$.

\section{THEORETICAL BACKGROUND}

\section{A. Key concepts of phenomenological and statistical thermodynamics}

In this paper, we are concerned with formation and thermodynamic stability of liquid-crystalline films adsorbed on solid surfaces. Therefore, it seems most appropriate to consider the grand canonical ensemble which permits relatively easy access to the grand potential $\Omega$. In the grand canonical ensemble

$$
\Omega\left(\mu, T, A, s_{\mathrm{Z}}\right)=-k_{\mathrm{B}} T \ln \Xi\left(\mu, T, A, s_{\mathrm{z}}\right),
$$

where $\mu$ denotes the chemical potential, $T$ is the temperature, $k_{\mathrm{B}}$ is the Boltzmann's constant,

$$
\Xi=\sum_{N}\left(\frac{\mathcal{I} \exp (\beta \mu)}{m \Lambda^{5}}\right)^{N} \mathcal{Z},
$$

is the partition function of the grand canonical ensemble $\left(\beta \equiv 1 / k_{\mathrm{B}} T\right)$, and

$$
\mathcal{Z}=\frac{1}{2^{N} N !} \int \mathrm{d} \boldsymbol{R} \mathrm{d} \widehat{\boldsymbol{U}} \exp (-\beta U),
$$

is the configuration integral. ${ }^{35}$ In Eq. (3.2), $\Lambda \equiv \sqrt{\beta h^{2} / 2 \pi m}$ is the thermal de Broglie wavelength, $m$ is the molecular mass, and $\mathcal{I}$ is the moment of inertia of a molecule. The prefactors $1 / 2^{N}$ and $N$ ! in Eq. (3.3) correct for double counting equivalent configurations that arise by replacing the set $\widehat{U}$ by the equivalent set $-\widehat{U}$ (see Sec. II) and take notice of the indistinguishability of the molecules, respectively. The quantity $U=U_{\mathrm{ff}}+U_{\mathrm{fs}}$ in the integrand is the total configurational potential energy of the liquid crystal [see Eqs. (2.1) and (2.7)]. that $^{35}$

By purely thermodynamic reasoning, ${ }^{40,41}$ one can show

$$
\mathrm{d} \Omega=-\mathcal{S} \mathrm{d} T-N \mathrm{~d} \mu-P_{\|} s_{\mathrm{z} 0} \mathrm{~d} A-P_{\mathrm{zz}} A_{0} \mathrm{~d} s_{\mathrm{z}},
$$

where $\mathcal{S}$ denotes entropy, $N$ is the number of molecules, and $P_{\|} \equiv \frac{1}{2}\left(P_{\mathrm{xx}}+P_{\mathrm{yy}}\right)$ and $P_{\mathrm{zz}}$ are the respective transverse and normal (diagonal) components of the pressure tensor $\mathbf{P}$ (with 
respect to the position of the substrate plane). In the absence of shear strains $\mathbf{P}$ can be represented by the $3 \times 3$ matrix

$$
\mathbf{P}=\left(\begin{array}{ccc}
P_{\|} & 0 & 0 \\
0 & P_{\|} & 0 \\
0 & 0 & P_{\mathrm{zz}}
\end{array}\right)
$$

In writing Eq. (3.4), we assume components of $\mathbf{P}$ to act on the faces of a rectangular fluid lamella where $A_{0}=s_{\mathrm{x} 0} s_{\mathrm{y} 0}$ such that $s_{\alpha 0}$ is the side length of the lamella in the direction of the $\alpha$-axis of a Cartesian coordinate system. ${ }^{40}$ Subscript " 0 " indicates that these side lengths refer to dimensions of the lamella in some unstrained reference state. Consequently, $A$ and $s_{\mathrm{z}}$ in Eq. (3.4) correspond to substrate area and separation but in a strained (i.e., compressed or dilated) state. This state can be realized in a gedankenexperiment by moving the boundaries separating the lamella from its surroundings. ${ }^{40,41}$ Hence, the last two terms in Eq. (3.4) account for mechanical work exchanged between the lamella and the surroundings.

The advantage of this approach is that $\Omega$ may be perceived as a homogeneous function of degree one in $A$ because $u_{\mathrm{fs}}$ depends only on the distance between a molecular centerof-mass and the substrate plane along the $z$-axis. Hence, $u_{\mathrm{fs}}$ is translationally invariant in both $x$ - and $y$-directions. ${ }^{40}$ One may therefore apply Euler's theorem and obtain

$$
\omega \equiv \frac{\Omega}{V}=-P_{\|},
$$

for the grand-potential density, where $V=A s_{\mathrm{z} 0}$ is the volume of the lamella. Employing the so-called hypervirial theorem, ${ }^{42}$ it follows from Eqs. (2.2) to (2.5) that ${ }^{35}$

$$
\begin{aligned}
P_{\alpha \alpha}= & \rho k_{\mathrm{B}} T-\frac{24}{V} / \sum_{(i, j)}\left[\left(\frac{\sigma}{r_{i j}}\right)^{12}-\frac{1}{2}\left(\frac{\sigma}{r_{i j}}\right)^{6}\right. \\
& \left.\left.\times \Psi\left(\widehat{\boldsymbol{r}}_{i j}, \widehat{\boldsymbol{u}}_{i}, \widehat{\boldsymbol{u}}_{j}\right)\right] \widehat{r}_{i j}^{\alpha} \widehat{r}_{i j}^{\alpha}\right\rangle \\
& -\frac{30 \varepsilon \varepsilon_{2}}{V}\left\langle\sum _ { ( i , j ) } ( \frac { \sigma } { r _ { i j } } ) ^ { 6 } \left\{\left(\widehat{\boldsymbol{u}}_{i} \cdot \widehat{\boldsymbol{r}}_{i j}\right) \widehat{u}_{i}^{\alpha} \widehat{r}_{i j}^{\alpha}\right.\right. \\
& +\left(\widehat{\boldsymbol{u}}_{j} \cdot \widehat{\boldsymbol{r}}_{i j}\right) \widehat{\boldsymbol{u}}_{j}^{\alpha} \widehat{r}_{i j}^{\alpha}-\left[\left(\widehat{\boldsymbol{u}}_{i} \cdot \widehat{\boldsymbol{r}}_{i j}\right)^{2}+\left(\widehat{\boldsymbol{u}}_{j} \cdot \widehat{\boldsymbol{r}}_{i j}\right)^{2}\right] \\
& \left.\left.\times \widehat{r}_{i j}^{\alpha} \widehat{r}_{i j}^{\alpha}\right\}\right\rangle, \quad \alpha=x, y .
\end{aligned}
$$

On the right side of Eq. (3.7), superscripts $\alpha$ refer to the $\alpha$ component of the associated vector and angular brackets denote a grand canonical ensemble average. The first term of the right side is the ideal-gas contribution where $\rho \equiv\langle N\rangle / V$ is the number density. Together with Eq. (3.6), Eq. (3.7) permits a quantitative discussion of phase equilibria. Additional properties of the grand-potential density are discussed in the Appendix.

\section{B. Properties}

Besides the grand-potential density already introduced in Eq. (3.6) measures of nematic order and structure of the adsorbed liquid crystals will be considered in this section. To that end, we introduce the so-called (instantaneous) alignment tensor $^{43}$

$$
\mathbf{Q}=\frac{1}{2 N} \sum_{i=1}^{N}\left(3 \widehat{\boldsymbol{u}}_{i} \otimes \widehat{\boldsymbol{u}}_{i}-\mathbf{1}\right),
$$

which is a second-rank tensor that can be represented by a $3 \times 3$ matrix. In Eq. (3.8), 1 denotes the unit tensor and the operator " $\otimes$ " stands for the direct (i.e., "dyadic") product. From Eq. (3.8), it is also clear that the alignment tensor is real and symmetric. Moreover, it is traceless such that $\operatorname{Tr} \mathbf{Q}=0$ where the operator " $\operatorname{Tr}$ " represents the trace. Using Jacobi's method, ${ }^{44}$ one may compute the eigenvalues $\lambda_{-} \leq \lambda_{0}$ $\leq \lambda_{+}$of $\mathbf{Q}$ and the associated eigenvectors. Following standard practice, ${ }^{35,36,45-49}$ we define the nematic (Maier-Saupe) order parameter as $S \equiv\left\langle\lambda_{+}\right\rangle$. The eigenvector associated with the instantaneous eigenvalue $\lambda_{+}$is taken as the nematic director $\widehat{\boldsymbol{n}}$. Moreover, $\mathbf{Q}$ may be diagonalized in the basis of its three eigenvectors to obtain $\operatorname{diag} \mathbf{Q}$. A basic theorem of linear algebra establishes the trace of a tensor as one of its scalar invariants ${ }^{50}$ such that $\operatorname{Tr} \mathbf{Q}=\operatorname{Tr}(\operatorname{diag} \mathbf{Q})=0$, where

$$
\operatorname{diag} \mathbf{Q}=\left(\begin{array}{ccc}
-\lambda_{+} / 2-\zeta & 0 & 0 \\
0 & -\lambda_{+} / 2+\zeta & 0 \\
0 & 0 & \lambda_{+}
\end{array}\right)
$$

which was demonstrated by Low who uses an expansion in terms of Wigner matrices. ${ }^{51}$ In Eq. (3.9), $\zeta$ is a measure of apparent biaxiality. As Eppenga and Frenkel already pointed out, $\zeta$ is nonzero in any finite system even in the isotropic phase. ${ }^{47}$ As we have recently demonstrated the associated ostensible biaxiality order parameter $\xi \equiv\langle\zeta\rangle$ may be useful in locating the isotropic-nematic (IN) phase transition in a reasonably large system. ${ }^{37}$

In addition to these global measures of order, it will also be interesting to determine order locally. To that end one notices that on account of the fluid-substrate interaction [see Eq. (2.7)], $\widehat{\boldsymbol{u}}_{i}$ in Eq. (3.8) may be perceived as a local variable $\widehat{\boldsymbol{u}}_{i}(z)$. This, of course, permits to define a local alignment tensor $\mathbf{Q}(z)$ such that a local nematic order parameter $S(z)$ $=\left\langle\lambda_{+}(z)\right\rangle$ may be introduced. However, some caution is advisable at this point. In computer simulations, $S(z)$ may be computed as a histogram by considering the orientations of $N(z)$ molecules located within a small volume $A \delta z$ centered on $z$. If this volume is relatively small so will be $N(z)$. Because of Eq. (2.2), these molecules tend to align their longer axes with some local director such that there is always some artificial local nematic order even though the entire liquid crystal is still in the isotropic phase. ${ }^{47}$ To correct for this finite-size effect Richter and Gruhn ${ }^{45}$ devised an efficient scheme that we employ throughout this work.

In addition, another measure of local order will turn out to be useful. Its definition is based upon the realization that the $z$-axis is a distinguished direction in our system due to the presence of the planar substrates. Hence, we define

$$
P_{2}(z)=\frac{1}{2}\left\langle\frac{1}{N(z)} \sum_{i=1}^{N(z)}\left\{3\left[\widehat{\boldsymbol{u}}_{i}(z) \cdot \widehat{\boldsymbol{e}}_{\mathrm{z}}\right]^{2}-1\right\}\right\rangle .
$$


From this definition, it is immediately clear that $P_{2}(z) \rightarrow-\frac{1}{2}$, if molecules are oriented in a parallel fashion with respect to the substrate plane; $P_{2}(z) \rightarrow 1$, if they are homeotropically aligned instead. In this latter case, $S(z)=P_{2}(z)$ whereas $S(z)$ and $P_{2}(z)$ will generally deviate as $P_{2}(z) \rightarrow-\frac{1}{2}$. Notice also that $S(z) \geq 0$, where the equality holds if and only if $\mathbf{Q}(z)=\mathbf{0}$, where $\mathbf{0}$ denotes the zero tensor. In this latter case, $\lambda_{-}=\lambda_{0}$ $=\lambda_{+}=0$ indicating an ideal isotropic arrangement of the molecules.

Finally, we introduce the local density of the fluid $\rho(z)$ as a measure of probability to find the center of mass of a molecule at a position $z$. Because of the form of the fluidsubstrate potential introduced in Eq. (2.7), the local density must be translationally invariant with respect to the $x$ - and $y$ components of the center of mass position $\boldsymbol{r}$. Formally, one may introduce $\rho(z)$ via the expression

$$
\rho(z) \equiv\left\langle\sum_{i=1}^{N} \delta\left(z-z_{i}\right)\right\rangle=\frac{\langle N(z)\rangle}{A \delta z},
$$

where $\delta\left(z-z_{i}\right)$ is the Dirac $\delta$-function and the far right side of the equation gives an operational expression useful for the computation of $\rho(z)$ in a computer simulation.

\section{RESULTS}

\section{A. Numerical details}

In this work, we employ MC simulations in the grand canonical ensemble employing the algorithm discussed in detail by Gruhn and Schoen. ${ }^{52}$ This algorithm closely resembles that proposed by Adams for a simple Lennard-Jones fluid. ${ }^{53}$ It allows one to realize numerically a Markov process that generates a distribution in configuration space proportional to $\exp \{-\beta[U(\boldsymbol{R}, \widehat{\boldsymbol{U}})-\mu N]-\ln N !-5 N \ln (\Lambda m / \mathcal{I})\}$ in agreement with Eqs. (3.2) and (3.3).

We refer to a $\mathrm{MC}$ cycle as a sequence of $N$ attempts to displace or rotate a molecule and $N$ attempted creations of new or removals of already existing molecules where $N$ is the actual number of molecules present at the beginning of a new cycle. To avoid biasing, the generation of configurations, displacements, and rotations as well as creation and removal are attempted with equal probability. Our simulations are based upon $2 \times 10^{4}$ cycles for equilibration followed by 2 $\times 10^{5}$ cycles to compute ensemble averages. As we have verified for a couple of thermodynamic states, these numbers are large enough to guarantee reliable results even in the immediate vicinity of a phase transition. To save computer time, we employ a combination of a conventional Verlet with a link-cell neighborlist as described in Allen and Tildesley's book.$^{54}$ In addition, fluid-fluid interactions are cut off beyond an intermolecular separation of $r_{\mathrm{c}}=3 \sigma$, which we use throughout this work; no such cutoff is applied to fluid-substrate interactions.

We begin a sequence of simulations at a sufficiently low $\mu$ at which a bulk system would be a thermodynamically stable, dilute gas. Subsequent runs are started from the final configuration of the preceding one where we increase $\mu$ in small steps $\delta \mu$. During this sequence, we monitor $\omega$ computed via
Eqs. (3.6) and (3.7). At some characteristic value of $\mu$, the system will become thermodynamically unstable and undergoes a transition from phase $i$ to some other phase $j$. However, this transition does not necessarily occur at coexistence, that is the associated grand-potential densities do not necessarily satisfy Eq. (A5) but the inequality $\omega_{j}<\omega_{i}$ instead. In other words, the transition typically takes the system from a metastable to a thermodynamically stable phase. To locate $\mu_{\mathrm{x}}^{i j}$ [see Eq. (A5)], one can start from the current state point in phase $j$ and decrease $\mu$ which causes the associated $\omega_{j}$ to increase because of Eq. (A1) until Eq. (A5) is satisfied.

This procedure is valid for phase transitions involving a sufficiently large density difference between phases $i$ and $j$ (for example, a transition from a gas-like film to a liquid-like phase). However, for reasons to be explained below, we are also interested in locating the IN phase transition which for the present model is accompanied by a rather minute density change. ${ }^{35,39}$ In this case, it will be useful to monitor the variation of the nematic order parameter $S$ with $\mu$.

Throughout this work, we express all quantities of interest in terms of the customary dimensionless (i.e., "reduced") units. For example, length is given in units of $\sigma$, energy in units of $\epsilon$, and temperature in units of $\epsilon / k_{\mathrm{B}}$. Other derived quantities are expressed in terms of suitable combinations of these basic quantities. For example, pressure-tensor elements are given in units of $\epsilon / \sigma^{3}$. In all the simulations, we consider a computational cell of $s_{\mathrm{x} 0}=s_{\mathrm{y} 0}=20, s_{\mathrm{z} 0}=50$ to guarantee that our model system effectively mimics a fluid phase interacting with a single substrate as we already demonstrated in Sec. II. Histograms of $\rho(z), S(z)$, and $P_{2}(z)$ are computed with a bin width $\delta z=0.1$. For this system size and over the range of chemical potentials studied between 900 and 17000 particles are accommodated on average at a constant temperature $T=0.9$ below the bulk gas-liquid critical point. ${ }^{34}$

\section{B. Phase transitions}

We begin our discussion of results with plots of $\omega$ versus $\mu$ in Fig. 3 for nh anchoring. For the shortest-range fluid-substrate potential characterized by $\eta=1.00$, the plot in Fig. 3(a) shows that a low-density phase is thermodynamically stable for $\mu \lesssim-13.00$. At this $\mu$, the plot of $\omega(\mu)$ for the low-density phase intersects another curve $\omega(\mu)$ with a slope significantly larger in magnitude corresponding to a higher-density phase [see Eq. (A1)]. The precise physical nature of these different phases requires an analysis of $\rho(z)$, $S(z)$, and $P_{2}(z)$, which we defer to Sec. IV C. Beyond the intersection (i.e., as $\mu$ increases further), the low-density phase remains metastable over a certain range of chemical potentials and apparently becomes unstable at that chemical potential at which the corresponding $\omega(\mu)$ curve ends. The same statements apply to $\omega(\mu)$ for the high-density phase below the chemical potential of intersection. If one increases the range of the fluid-substrate interaction potential to $\eta$ $=0.45$, plots in Fig. 3(b) show that a third phase of intermediate density arises such that two chemical potentials exist at which pairs of phases coexist. Increasing the range of fluid-substrate interactions by decreasing the screening 


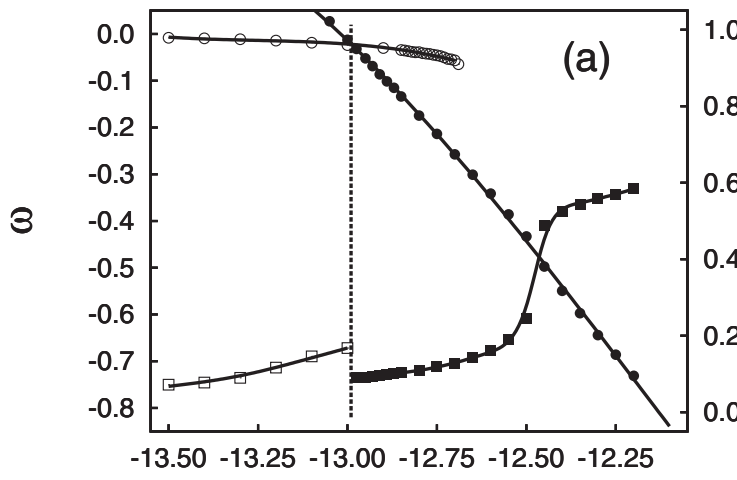

$\mu$

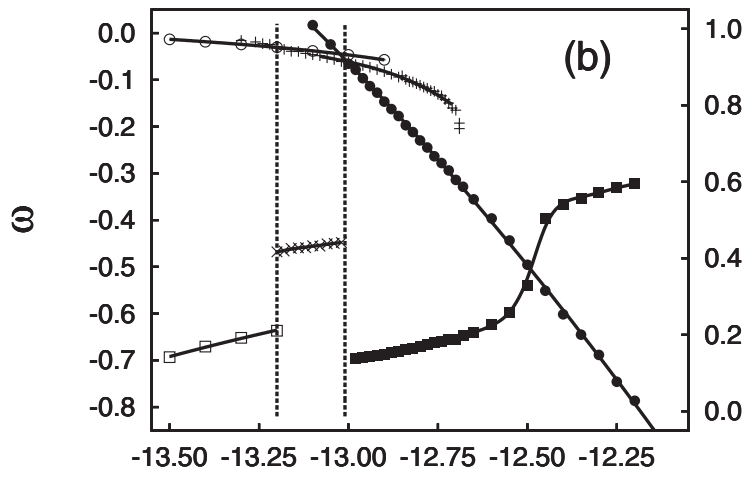

$\mu$

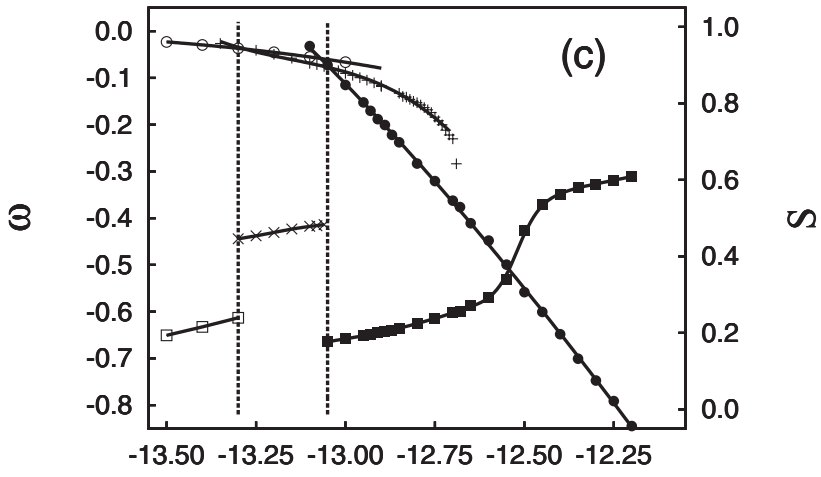

$\mu$

FIG. 3. Plots of grand-potential density $\omega$ and (global) nematic order parameter $S$ as functions of chemical potential $\mu$ for nh anchoring (see Table I). (a) $\eta=1.00$ [see Eq. (2.7)], (०) $\omega$, ( $\square$ ) $S$ for a gas-like adsorbed film and (•) $\omega$, (ם) $S$ for a liquid-like phase. (b) As (a) but for $\eta=0.45$; notice that a prewetting transition occurs where $(+)$ represents $\omega$ and $(\times)$ refers to $S$. (c) As (b) but for $\eta=0.25$. Vertical dashed lines demarcate limits of thermodynamic stability for specific phases whereas solid lines are fits to the discrete data points to guide the eye.

constant to $\eta=0.25$, the plot in Fig. 3(c) reveals that the one-phase region for the intermediate-density phase shifts to slightly lower chemical potentials and widens somewhat.

The corresponding plot of the global nematic order parameter $S$ in Fig. 3(a) indicates that with increasing chemical potential nematic order increases slightly in the low-density phase but remains relatively small even at phase coexistence. Hence, the low-density phase remains more or less isotropic globally. At phase coexistence, $S$ drops slightly and discontin-

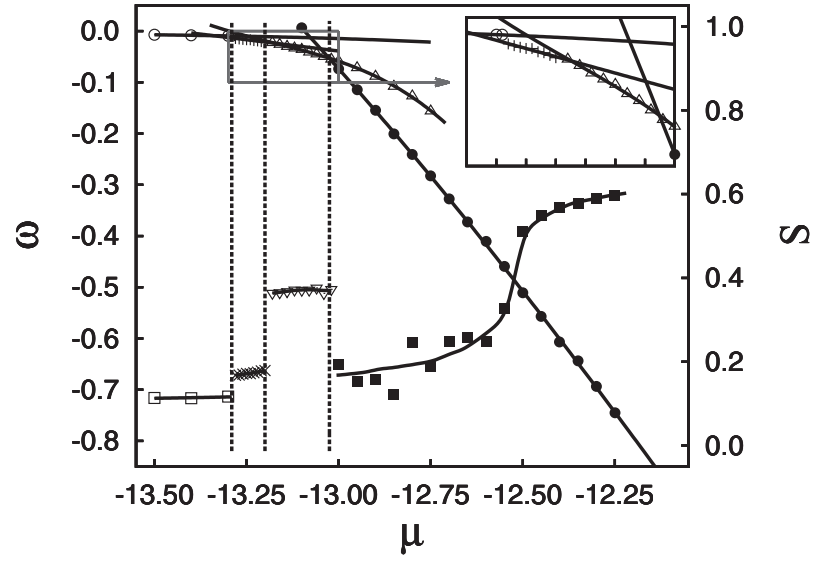

FIG. 4. As Fig. 3(c), but for the pd anchoring scenario (see Table I). Inset is an enlargement of the part of the plot within the box.

uously indicating that the newly formed high-density phase is more isotropic. Interestingly, when the intermediate-density phase forms in Figs. 3(b) and 3(c), nematic order increases substantially reflected by an increase of $S$ by roughly a factor of 2 . When the intermediate-density phase gives way to the high-density phase, this high nematic order is lost at first. However, in all parts of Fig. 3, one realizes that eventually there is a strong increase in nematic order at sufficiently high $\mu \simeq-12.50$. This value of $\mu$ is almost independent of the range of the fluid-substrate interaction potential indicating that nematization is mostly occurring in the bulk of the high-density phase, that is in regions that are not subject to strong fluid-substrate interactions. However, the shape of the plot of $S$ versus $\mu$ becomes a bit more rounded as $\eta$ decreases.

The formation of the nematic phase is accompanied by a small change in mean density of the liquid crystal. ${ }^{35} \mathrm{Be}$ cause of Eqs. (A1) and (A3), one would therefore anticipate a change both in slope and curvature in the plots of $\omega(\mu)$ at that $\mu$ at which the IN phase transition occurs. However, this effect seems to be much smaller than the statistical accuracy with which the curves $\omega(\mu)$ in Fig. 3 can be obtained.

These general features are retained if the pd instead of the nh anchoring scenario is considered. Therefore, to save space we show results obtained for the pd anchoring only for the case $\eta=0.25$ in Fig. 4 as a typical illustration. The most significant difference between the plots in Fig. 4 and the corresponding Fig. 3(c) concerns the existence of four instead of three phase transitions over the range of chemical potentials considered. Increasing $\mu$ from the smallest value for which data have been plotted in Fig. 4, a low-density phase of nearly constant $\omega$ is thermodynamically stable until $\mu \simeq-13.28$. Over this range of $\mu$ 's, the corresponding plot of $S$ shows that there is relatively little nematic order in our system. At $\mu \simeq-13.28$, two of the curves $\omega(\mu)$ of slightly different slope intersect such that a phase of slightly larger mean density is thermodynamically stable for $\mu \gtrsim-13.28$. This new phase exhibits a somewhat enhanced degree of nematic order but $S$ $<0.2$ still remains relatively small. At $\mu \simeq-13.18$ a second discontinuous phase transition arises. From the inset in Fig. 4, one notices that in the newly formed phase the magnitude of the slope of $\omega(\mu)$ is again larger. Hence, this new 

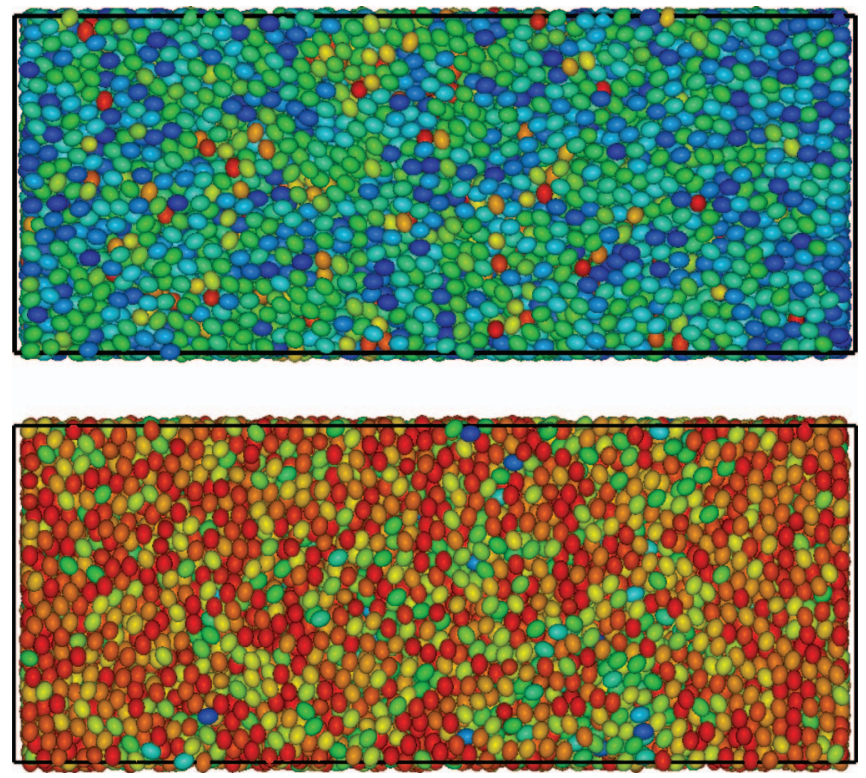

FIG. 5. "Snapshots" from MC simulations in the $x-z$ plane. The solid substrates (not shown) are at the left and right ends of the rectangular slabs, respectively. The black solid line represents the simulation box. The snapshots correspond to $\eta=0.25$ and $\mu=-12.25$, where the system in both nh (upper plot) and pd anchoring scenarios (lower plot) is deep in the nematic phase [see Figs. 3(c) and 4]. Choosing the horizontal direction (z-axis) particles are colored in blue if $\widehat{\boldsymbol{u}}_{i} \cdot \widehat{\boldsymbol{e}}_{\mathrm{z}}=1$, whereas particles in red satisfy $\widehat{\boldsymbol{u}}_{i} \cdot \widehat{\boldsymbol{e}}_{\mathrm{z}}=0$.

phase is characterized by an elevated mean density compared with its immediate predecessor at lower $\mu$. An inspection of the corresponding data for $S$ shows that nematic order increases quite substantially at the second phase transition but remains smaller than $S=0.4$ over the range of $\mu$ 's for which this phase remains thermodynamically stable. This range ends at $\mu \simeq-13.02$ such that for $\mu \gtrsim-13.02$ a high-density phase characterized by the largest magnitude of the slope of $\omega(\mu)$ is thermodynamically stable. When this phase forms at $\mu \simeq-13.02, S$ drops by almost a factor of 2 such that this high-density phase is mostly isotropic.

Eventually, at $\mu \simeq-12.50$ an IN phase transition occurs and $S$ rises steeply as $\mu$ increases further. A comparison with plots in Fig. 3(c) shows that the IN phase transition again occurs at about the same $\mu$ irrespective of the anchoring scenario. However, the direction of $\widehat{\boldsymbol{n}}$ is determined by the specific anchoring as plots in Fig. 5 illustrate. The upper plot in Fig. 5 is a representative "snapshot" from the MC simulation illustrating the structure of the nematic phase for the nh anchoring scenario. As one can see molecules are mostly homeotropically aligned with a considerable number of defects (i.e., particles with orientations other than those imposed by the specific anchoring scenario). Because the fluid-substrate potential at the center of the simulation box has approximately decayed to zero (see Fig. 2) the dominant homeotropic alignment is mediated by molecules that are closer to the substrate. Hence, it is possible to "imprint" the chemical nature of the substrates represented by a specific anchoring scenario onto an adjacent liquid-crystalline phase through a collective effect and over relatively large distances. A more quantitative discussion of local structural features of nematic phases and different anchoring scenarios is, however, postponed until Sec. IV C.

One also notices from Figs. 3(c) and 4 that the scatter of $S$ in the high-density isotropic phase $(-13.00 \leq \mu \leq-12.5)$ is substantially larger for the pd compared with the nh anchoring scenario which we rationalize as follows. At the lower substrate the anchoring function $g^{[1]}(\widehat{\boldsymbol{u}})$ is degenerate in the sense of Jerôme ${ }^{1}$ because molecules do not suffer any energy penalty as long as they align their long axes with any direction on the unit circle in the $x-y$ plane. On the other hand, $g^{[2]}(\widehat{\boldsymbol{u}})$ causes monostable (d) anchoring. If $S$ is relatively small, only molecules in the immediate vicinity of the substrate exhibit a certain degree of local nematic order with associated local directors $\widehat{\boldsymbol{n}}^{[1]}(z)$ and $\widehat{\boldsymbol{n}}^{[2]}(z)$. These local nematic regions are separated by a relatively large isotropic regime. Because of its weakness, local nematic order is short range. Due to monostable anchoring, $\widehat{\boldsymbol{n}}^{[2]}(z)$ remains more or less constant. On the contrary, $\widehat{\boldsymbol{n}}^{[1]}(z)$ may vary in the $x-y$ plane because of the degenerate planar anchoring. Whereas this does not affect the local degree of nematic order, any angle between $\widehat{\boldsymbol{n}}^{[1]}(z)$ and $\widehat{\boldsymbol{n}}^{[2]}(z)$ affects the global order parameter computed via $\mathbf{Q}$ which involves a sum over all molecular orientations [see Eq. (3.8)]. This argument also implies that once the system is in the nematic phase, molecules local and global directors become fixed in space such that the scatter in $S$ should be smaller. Indeed, our results for $\mu \gtrsim-12.50$ displayed in Fig. 4 comport with this line of arguments. Moreover, we realize that none of the plots of $S$ in Fig. 3 suffers from similar statistical accuracies in the isotropic high density regime. This is because at the substrate molecules align always homeotropically regardless of whether they are anchored nonspecifically or homeotropically. ${ }^{35,36}$ In these cases, similar reorientation effects are therefore prevented by both anchoring functions.

\section{Structure and local orientation}

The question now becomes: what is the physical nature of the different phases represented by the plots in Fig. 3? This question may be addressed directly by considering $l o-$ cal structure and orientation of the various phases. We begin discussing these features in Fig. 6 for the shortest-range fluid-substrate potential characterized by $\eta=1.00$. As the corresponding plots in Fig. 3(a) indicate two phases coexist at $\mu \simeq-13.00$. Corresponding plots of $\rho(z)$ in Fig. 6(a) show that the lower-density phase consists essentially of a thin film of three fluid layers at the nonspecifically anchoring, lower substrate $\left(z / s_{\mathrm{z} 0}=-0.5\right)$ whereas only a monolayer of very low density is adsorbed at the homeotropically anchoring upper substrate $\left(z / s_{\mathrm{z} 0}=+0.5\right)$. These layers are reflected by periodic maxima of $\rho(z)$. As we have verified, lowering $\mu$ causes molecules to desorb continuously from the nonspecifically anchoring substrate until only a submonolayer film is left.

Corresponding plots of both $S(z)$ and $P_{2}(z)$ reveal that molecules are homeotropically aligned where the degree of homeotropic alignment is larger at the upper substrate as one would have anticipated. At the nonspecifically anchoring, lower substrate one sees that $S(z) \approx P_{2}(z)$ as far as the first three layers are concerned. However, as one approaches 

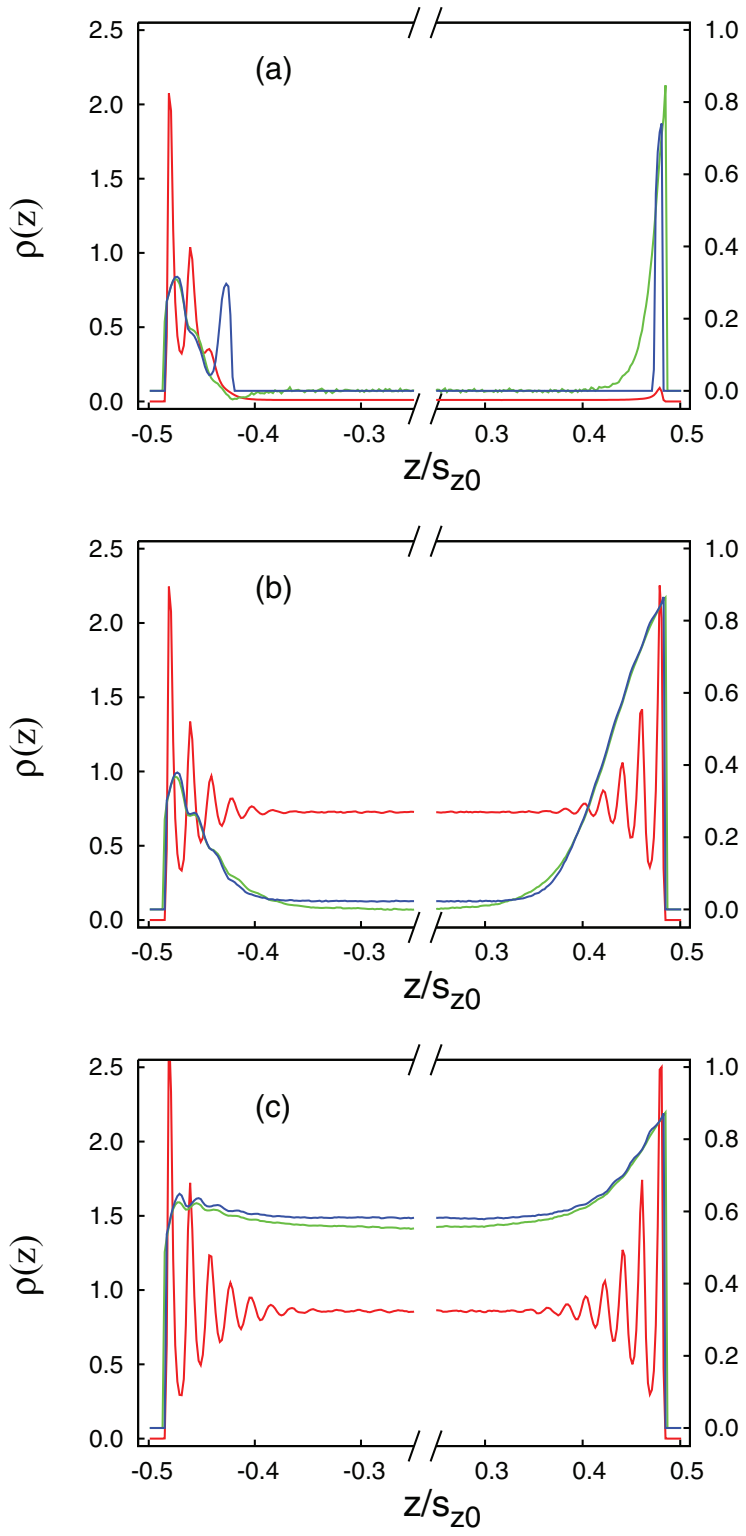

FIG. 6. Plots of local density $\rho(z)$ (一) and local orientational order parameters $P_{2}(z)(-)$ and $S(z)(-)$ as functions of position $z$ between lower $\left(z / s_{\mathrm{z} 0}\right.$ $=-0.5)$ and upper substrate $\left(z / s_{\mathrm{z} 0}=+0.5\right)\left(s_{\mathrm{z} 0}=50\right)$. Data are obtained for nh anchoring and $\eta=1.00$. Plots in parts (a) and (b) have been obtained for the same chemical potential $\mu=-13.00$ and correspond to coexisting phases of lower and higher mean density, respectively. The plots in part (c) have been obtained at $\mu=-12.20$ deep in the nematic high-density phase [see Fig. 3(a)].

the interface between the thin film and the adjacent bulk-like gas phase $S(z) \neq P_{2}(z)$ indicating that molecules prefer to align themselves parallel with the substrate plane. The preferred alignment at the film-gas interface is signaled by a pronounced peak in $S(z)$ at about $z / s_{z 0} \simeq-0.42$ in Fig. 6(a). Note also that this peak in $S(z)$ is not an artifact caused by the relatively small number of molecules located at the film-gas interface because our data have been corrected for such finite-size effects as we explained in Sec. III B.

The structure of the coexisting phase can be analyzed through the plots displayed in Fig. 6(b). Comparing these latter plots with the ones in Fig. 6(a), one notices that the liquid crystal has a bulk-like, homogeneous region where $\rho(z)=\rho_{\mathrm{b}}$
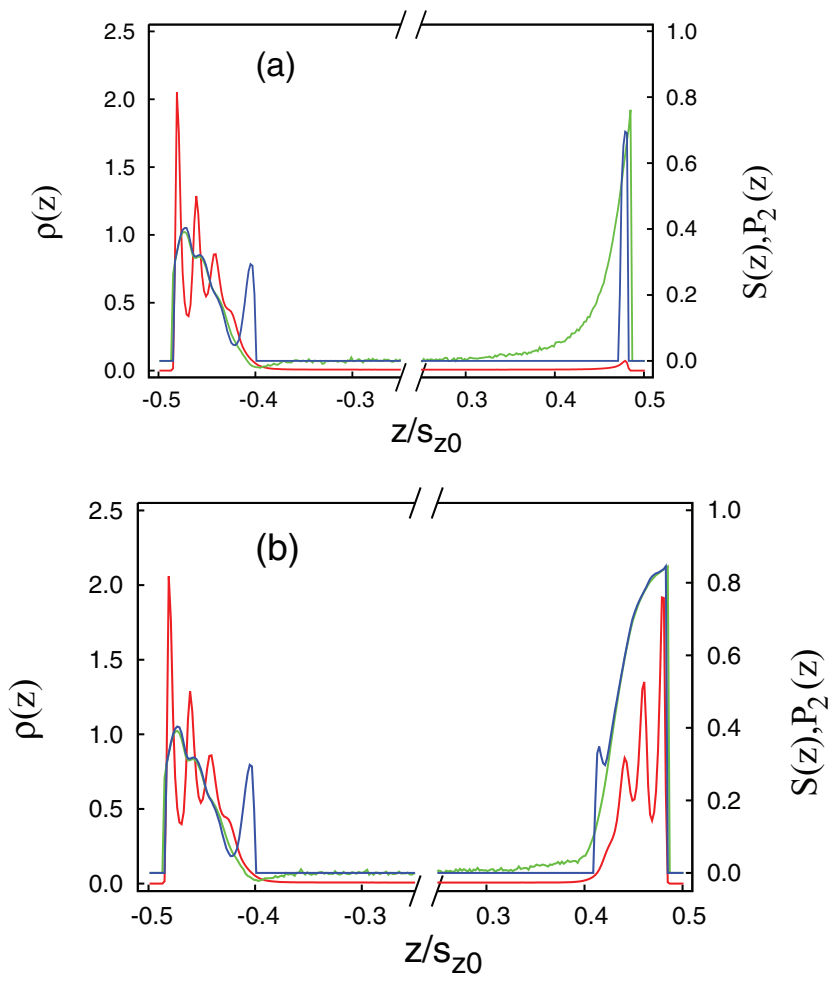

FIG. 7. As Fig. 6, but for $\eta=0.25$. Plots in parts (a) and (b) have been obtained for the same chemical potential $\mu=-13.30$ and correspond to coexisting phases of lower and intermediate mean density, respectively [see Fig. 3(c)].

and $\rho_{\mathrm{b}}$ is the density of a corresponding bulk liquid crystal at the same $T$ and $\mu$. Closer to each substrate the molecules arrange their centers of mass in individual layers parallel with the substrate plane. At the nonspecifically anchoring substrate, nematic order remains relatively low compared with the homeotropically anchoring substrate. As one departs from the substrate planes, nematic order decays such that the bulklike region of the liquid crystal remains isotropic. In the more ordered portions close to the substrates, molecules are homeotropically aligned as revealed by the relation $P_{2}(z)$ $\simeq S(z)$. Notice also that in Fig. 6(b), the specific anchoring scenario affects only the orientation of the molecules but not their packing. This is inferred from the plot of $\rho(z)$ in Fig. 6(b) which, unlike its counterpart in Fig. 6(a), remains symmetric with respect to the midplane located at $z=0$.

If the fluid-solid interaction potential becomes longer range plots in Figs. 3(b) and 3(c) indicated that an intermediate-density phase arises which is accompanied by a sudden increase in global nematic order. Plots in Figs. 7 and 8 illustrate the structure of the coexisting phases at $\mu=$ -13.30 and -13.05 and for $\eta=0.25$. As shown before in Fig. 6(a), the corresponding plot in Fig. 7(a) shows that a film of three distinct layers is adsorbed at the nonspecifically anchoring substrate whereas a very dilute monolayer is stabilized by the homeotropically anchoring substrate. Orientational effects in Fig. 7(a) are also very similar to those visible from the corresponding plots in Fig. 6(a). This applies in particular to the maximum in $S(z)$ at the film-gas interface located approximately at $z / s_{z 0} \simeq-0.4$. However, the corresponding 


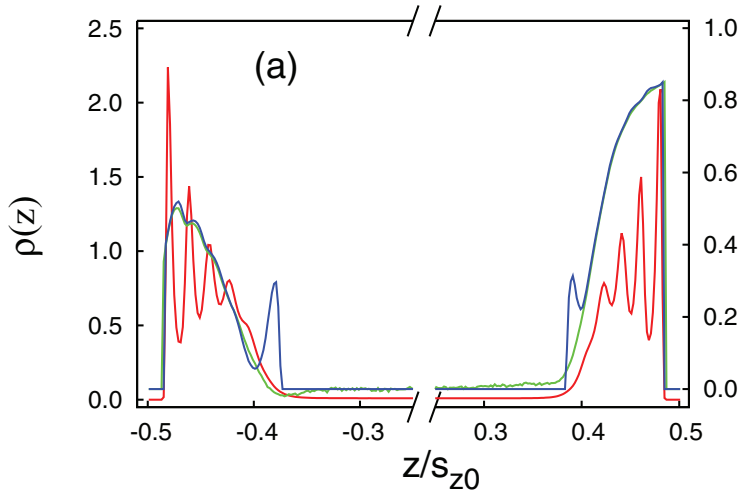

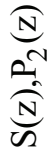

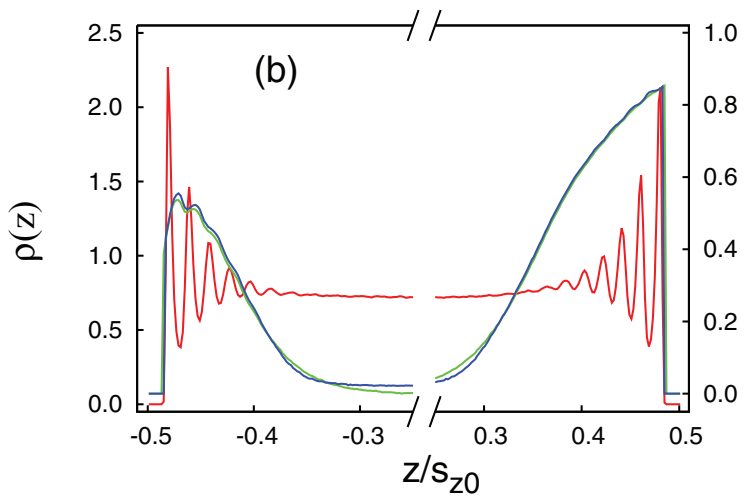

FIG. 8. As Fig. 6, but for $\eta=0.25$. Plots in parts (a) and (b) have been obtained for the same chemical potential $\mu=-13.05$ and correspond to coexisting phases of higher and intermediate mean density, respectively [see Fig. 3(c)].

plot in Fig. 7(b) reveals that at the homeotropically anchoring substrate the adsorbed film has undergone a prewetting transition from essentially a thin submonolayer to a microscopically thicker film of three distinct layers. This thicker film also exhibits distinct nematic order because the condition $P_{2}(z)=S(z)$ is satisfied until the film-(bulk) gas interface is reached. Its degree of nematic order is substantially larger than that observed for the film adsorbed at the opposite, nonspecifically anchoring substrate as one would have anticipated. However, these directions are slightly different for the film adsorbed at the nonspecifically and that at the homeotropically anchoring substrate. As one can see from the corresponding plots of $P_{2}(z)$, this quantity passes through a weak minimum when $S(z)$ becomes maximum at the interface at the nonspecifically anchoring substrate. At the homeotropically anchoring substrate, both $S(z)$ and $P_{2}(z)$ remain positive at the interface. Similar features have been reported earlier at the nematic-gas interface of homogeneous, free-standing liquid crystal films. ${ }^{55,56}$ Hence, as far as nh anchoring is concerned an already ordered submonolayer film undergoes a prewetting transition to form a molecularly thick nematic film.

As the chemical potential increases over the range $-13.30 \leq \mu \leq-13.05$, the films adsorbed at both substrates increase in thickness. This can be seen by comparing plots in Figs. 7(b) and 8(b) which show that for both films the film-gas interface has shifted to smaller values of $|z|$. Notice that at both substrates, one additional layer of molecules is visible in the plot of $\rho(z)$ in Fig. 8(b) compared with the corresponding one in Fig. 7(b). At $\mu=-13.05$, the gas phase separating the adsorbed films at both substrates condenses spontaneously such that again a bulk-like, homogeneous fluid forms as the plot in Fig. 8(b) shows. Comparing Fig. 8(b) with the corresponding plot in Fig. 6(b), one notices that nematic order induced by both substrates is much more pronounced if the fluid-substrate interaction is longer range. Moreover, as one approaches the midplane at $z=0$, the preferential orientation of the molecules extends much farther into the bulk-like region of the liquid crystal. One also notices that in this bulklike region $\rho(z)$ does not correspond to $\rho_{\mathrm{b}}$ but decays slightly towards the midplane. This is because the slow decay of orientational order as one departs from either substrate causes the packing of molecules to gradually change. This effect has been analyzed earlier by us ${ }^{36}$ for the same model liquid crystal but for molecules with a slightly larger aspect ratio.
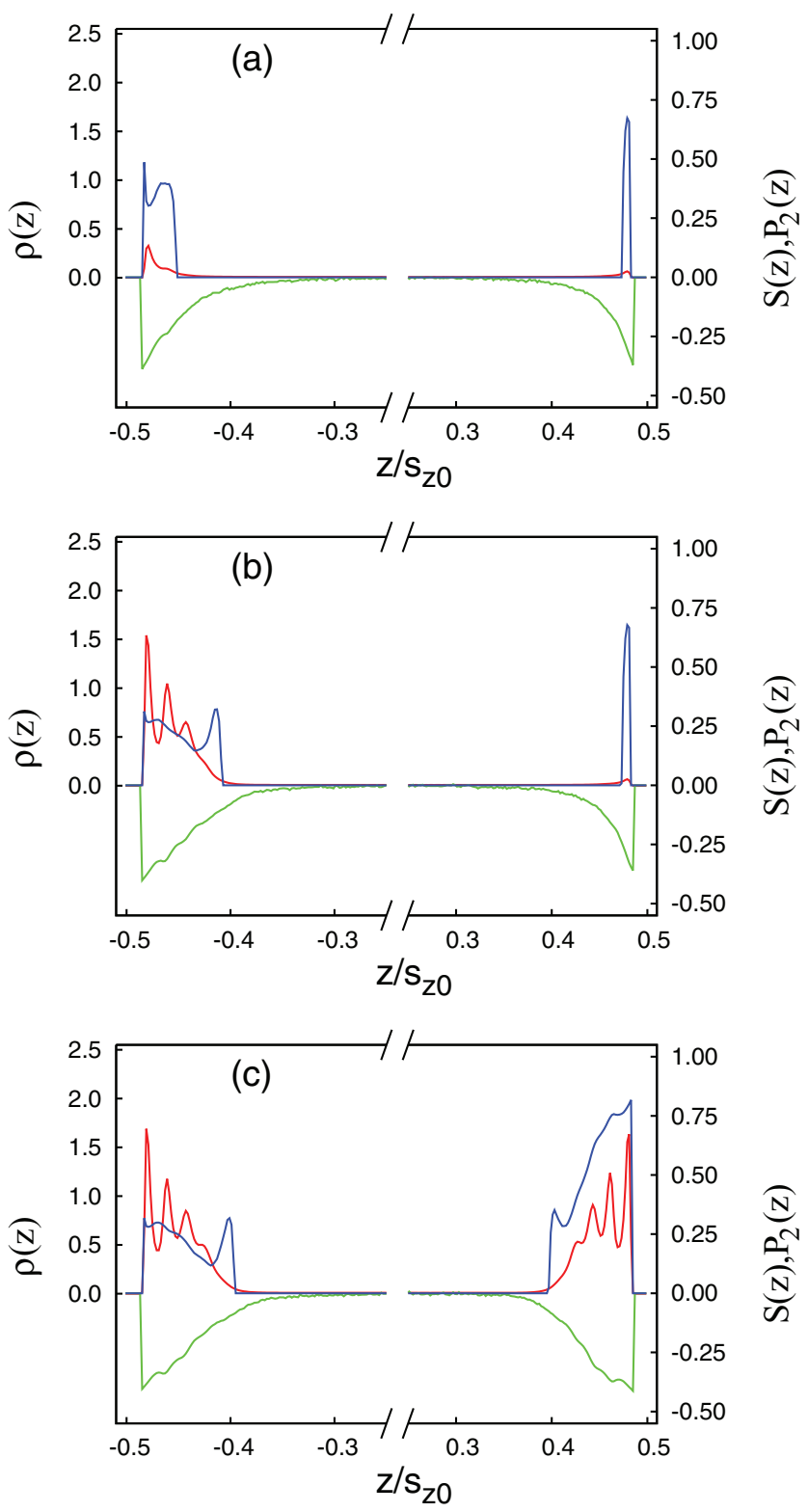

FIG. 9. As Fig. 7, but for the pd anchoring scenario. (a) $\mu=-13.29$, (b) $\mu$ $=-13.28$, and (c) $\mu=-13.18$ (see also Fig. 4). 
Finally, one notices from Fig. 3(c) that at sufficiently large $\mu$, the global order parameter for the high-density, liquid-like system increases. This indicates again that the liquid crystal undergoes an IN phase transition. The structure of the nematic phase at $\eta=0.25$ turns out to be very similar to what has already been illustrated by the plots in Fig. 6(c) for the shorter-range fluid substrate potential $(\eta=1.00)$. In the interest of conciseness, we therefore refrain from presenting corresponding data for $\eta=0.25$.

At this stage, it seems interesting to investigate the structure of coexisting phases for the pd anchoring scenario. We are again restricting this discussion to the longest-range fluid substrate potential $(\eta=0.25)$. At sufficiently low $\mu$ $\lesssim-13.28$, thin submonolayer films are adsorbed at both substrates [see Fig. 9(a)]. Here, molecules have a preferred parallel orientation with respect to the substrate planes as expected. This is inferred from a relatively large value of $S(z)$ whereas $P_{2}(z)$ remains negative. The submonolayer adsorbed at the lower, parallel anchoring substrate is in equilibrium with a thicker film comprising roughly four layers of molecules. Hence, at $\mu \simeq-13.28$, a prewetting transition occurs at the planar anchoring substrate [see Fig. 9(b)]. The submonolayer film adsorbed at the directionally anchoring upper substrate remains thermodynamically stable until at $\mu \simeq-13.18$, a second prewetting transition occurs during which the monolayer at the directionally anchoring substrate is transformed into a thicker film of about four distinct layers as the plots in Fig. 9(c) illustrate. Again, molecules pertaining to these films exhibit a preferred planar orientation with respect to the substrate plane. As $\mu$ increases, the films at both substrates grow in thickness continuously until at $\mu \simeq-13.02$, the entire
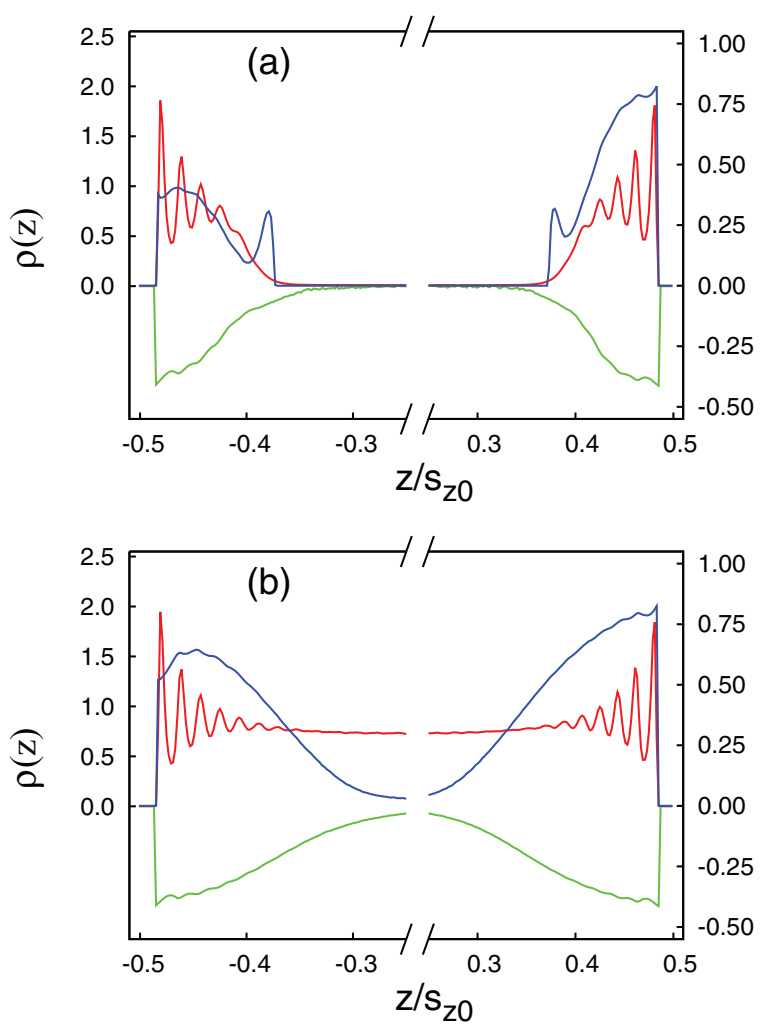

FIG. 10. As Fig. 9, but for $\mu=-13.02$ (a) and $\mu=-13.00$ (b).

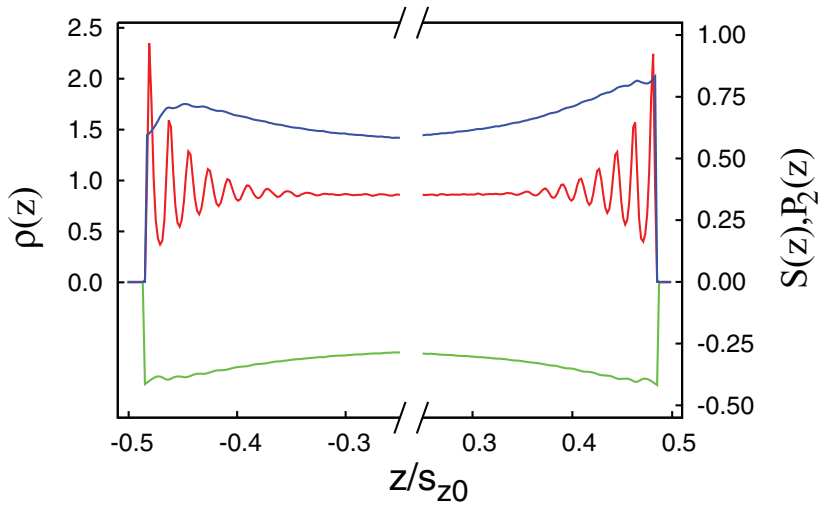

FIG. 11. As Fig. 9, but for $\mu=-12.25$ (see also Fig. 4).

system condenses and a mostly isotropic, high-density phase forms (see Fig. 4).

A comparison of plots of $S(z)$ in Figs. 9(c) and 10(a) show that at the lower, planar aligning substrate, the order of the adsorbed film increases only slightly with $\mu$. At the upper, directionally aligning substrate, the degree of local order remains nearly the same as the film thickens. Local nematic order at the lower substrate increases only in the presence of a stabilizing isotropic liquid as a comparison between plots in Figs. 10(a) and 10(b) reveals. This observation is in contrast with findings by Rull et al. who observed surface nematization in a thin film without the presence of an adjacent isotropic liquid. ${ }^{57}$ However, we would see an effect similar to the one reported by Rull et al. but only for already metastable nematic films.

Increasing $\mu$ further causes an IN phase transition in the high-density phase at $\mu \simeq-12.50$ as the corresponding plots in Fig. 4 clearly show. The structure of this high-density nematic phase is illustrated by the plots in Fig. 11. As one can see there molecules in the entire system have a preferred planar orientation with the solid substrates because $S(z)$ is large whereas $P_{2}(z)$ is negative. The structure of the nematic phase is also illustrated by the "snapshot" from an MC simulation presented as the lower part of Fig. 5.

\section{DISCUSSION AND CONCLUSIONS}

In this work, we employ MC simulations in the grand canonical ensemble to study wetting of solid substrates by a model liquid crystal. We focus on different anchoring scenarios in which specific orientations of the molecules are energetically discriminated. We consider two types of anchoring scenarios that can be described as "degenerate" and "monostable". Anchoring scenarios pertaining to the latter group (i.e., homeotropic and directional anchoring) favor orientations of molecules along a single easy axis whereas those scenarios belonging to the former class give molecules the freedom to orient themselves either in all three spatial directions (i.e., nonspecific anchoring) or in any direction within the substrate plane (i.e., planar anchoring) without an energy penalty.

The attractive part of the fluid-substrate potential is modeled via a Yukawa potential. This has the advantage that by 
varying the screening parameter, we are able to gradually change the range of the fluid-substrate attraction. To compare data for various screening parameters, we introduce two additional parameters that allow us to fix both the position of the minimum of the fluid-substrate potential and the value of the potential at the position of that minimum. For purely technical reasons, we "confine" the liquid crystal to a slit-pore. However, we make the distance between the pore walls sufficiently large so that molecules do not interact simultaneously with both walls. In effect, this permits us to study orientation effects in liquid-crystalline materials wetting single solid surfaces.

We consider a single temperature $T=0.9$ which is both below the bulk gas-(isotropic) liquid critical point and above the gas-isotropic-nematic triple point. The latter is inferred from the fact that with increasing $\mu$, the transition to the isotropic liquid and eventually from that phase to the nematic are well separated in the bulk. This is concluded from plots in Figs. 3 and 4, which show that both transitions occur at about the same $\mu$ irrespective of the specific anchoring scenario. In other words, the location of these transitions is unaffected by the presence of the substrates.

Our key findings can be summarized as follows. Substrates with degenerate anchoring are always covered with a submolecularly thin film at sufficiently low $\mu$. Increasing $\mu$ further causes the thickness of this film to increase continuously until a thick film consisting of a few molecular layers has formed. If anchoring is degenerate but results in planar orientation of the adsorbed molecules, a prewetting transition occurs at which a submonolayer film is transformed discontinuously into a molecularly thick film. A prewetting transition is also observed for the two monostable ( $\mathrm{d}$ and $\mathrm{h}$ ) anchoring scenarios considered in this study. In this latter case, $\mu_{\mathrm{x}}^{i j}$ is roughly the same for both $\mathrm{d}$ and $\mathrm{h}$ anchoring and exceeds $\mu_{\mathrm{x}}^{i j}$ for $\mathrm{p}$ anchoring significantly.

Anchoring is associated with orientational configurational entropy. This can be realized by comparing degenerate with monostable anchoring. Specifically, for $\mathrm{n}$ anchoring, where no orientation of a molecule with respect to the substrate plane receives any energy penalty, more configurations of molecules are possible than for both $\mathrm{d}$ and $\mathrm{h}$ anchoring. Because no molecular orientation is preferred by $\mathrm{n}$ anchoring, one might also consider this anchoring scenario as weak. In these latter cases, all orientations except for a single one receive such an energy penalty to a larger or lesser extend depending on the degree of misalignment of molecules with the easy axis. Moreover, comparing $n$ with $\mathrm{p}$ anchoring, orientational configurational entropy should be larger for the former because for the latter anchoring scenario only those orientations that lie on the unit circle in the $x-y$ plane avoid receiving an energy penalty. Orientational configurational entropy is therefore expected to decrease in the direction $\mathrm{n} \rightarrow \mathrm{p} \rightarrow \mathrm{d}$, h. Because no prewetting occurs at $\eta=0.25$ for $n$ anchoring, a prewetting transition is observed for $\mathrm{p}$ anchoring, and because $\mu_{\mathrm{x}}^{i j}$ is smaller for $\mathrm{p}$ compared with both $\mathrm{d}$ and $\mathrm{h}$ anchoring, we conclude that prewetting is shifted to lower $\mu$, the larger the orientational configurational entropy is. This interpretation is further corroborated by the observation that $\mu_{\mathrm{x}}^{i j}$ is roughly the same for the two monostable anchoring scenarios which are expected to give rise to a similar orientational configurational entropy. Hence, because of the close relationship between orientational effects caused by different anchoring and the existence/location of the prewetting transition, we refer to this phase transition as "orientational prewetting." Hence, we conclude that orientational prewetting is caused by substrates reducing the orientational configuration entropy of an adjacent sufficiently thin liquid-crystalline film. As $\mu$ increases beyond its value at the prewetting transition, the films at both substrates (mono- and multistable anchoring) continue to grow in thickness until finally complete wetting sets in.

Whether or not a thermodynamically stable prewetting film forms depends on the range of the fluid-substrate attraction, i.e., whether one is above or below the prewetting critical temperature which is expected to depend on the fluidsubstrate attraction. These findings are qualitatively similar to the ones reported earlier by Telo da Gama using densityfunctional theory at mean-field level. ${ }^{20}$ Here we find that for the shortest-range attraction $(\eta=1.00)$, orientational prewetting does not occur. Instead, the monolayer film adsorbed on the monostable anchoring substrate vanishes during a wetting transition when the entire liquid crystal between both substrates condenses (complete wetting). The new liquid-like phase is mostly isotropic except for the first few layers adsorbed on the substrate which retain a certain degree of local nematic order. This situation may be perceived as one in which a substrate-controlled nematic region is completely wet by an isotropic liquid. The decay of nematic order with increasing distance from the substrate is closely correlated with the range of fluid-solid attraction.

At a sufficiently high $\mu$, the entire liquid crystal undergoes an IN phase transition. The location of the IN phase transition is nearly independent of the precise anchoring scenario as one would expect if the distance between the solid substrates is sufficiently large as we have argued above. However, both "snapshots" of characteristic configurations of molecules as well as properly defined orientation profiles show that the specific anchoring scenario has a significant impact on $\widehat{\boldsymbol{n}}$. Hence, even though the range of direct fluid-substrate interaction is finite, the specific anchoring scenario is imprinted on more remote portions of the liquid crystal (i.e., portions farther removed from the solid substrate) through mediation by molecules that are closer to the substrate.

\section{ACKNOWLEDGMENTS}

We are grateful for financial support from the International Graduate Research Training Group 1524 "SelfAssembled Soft Matter Nanostructures at Interfaces.”

\section{APPENDIX: PROPERTIES OF THE GRAND-POTENTIAL DENSITY}

Here we introduce additional properties of the grandpotential density that are useful in analyzing the phase behavior of fluid phases. First, one notices from Eqs. (3.4) and (3.6) that

$$
\left(\frac{\partial \omega_{i}}{\partial \mu}\right)_{\{\cdot\} \backslash \mu}=-\rho_{i}<0,
$$


where " $\{\cdot\} \backslash \mu$ " is the shorthand notation to indicate that upon differentiation all other natural variables of $\omega$ except for $\mu$ are to be held constant. Subscript $i$ refers to the specific thermodynamic phase under consideration. Because the number density is a positive definite quantity by definition, we anticipate plots of $\omega_{i}$ versus $\mu$ at constant $T, A$, and $s_{\mathrm{Z}}$ to be monotonically decreasing where the slope is the larger the higher $\rho_{i}$ is for a given phase. Second, using the definition $\kappa_{\|} \equiv-A^{-1}\left(\partial A / \partial P_{\|}\right)_{T, N, s_{\mathrm{z}}}$ for the transverse isothermal compressibility together with the Gibbs-Duhem equation ${ }^{40}$

$$
0=\mathcal{S} \mathrm{d} T+N \mathrm{~d} \mu-A s_{\mathrm{z} 0} \mathrm{~d} P_{\|}+P_{\mathrm{zz}} A_{0} \mathrm{~d} s_{\mathrm{z}},
$$

one can easily verify that at constant $T$ and $s_{\mathrm{z}}{ }^{40}$

$$
\left(\frac{\partial^{2} \omega_{i}}{\partial \mu^{2}}\right)_{\{\cdot\} \backslash \mu}=-\rho_{i}^{2} \kappa_{\|}<0,
$$

which holds because thermodynamic stability requires $\kappa_{\|}$ $>0 .{ }^{58}$ Hence, for $T=$ constant, the functions $\omega_{i}(\mu)$ are concave, that is the inequality

$$
\omega_{i}(\mu) \geq \frac{\omega_{i}\left(\mu^{\prime \prime}\right)\left(\mu-\mu^{\prime}\right)+\omega_{i}\left(\mu^{\prime}\right)\left(\mu^{\prime \prime}-\mu\right)}{\mu^{\prime \prime}-\mu^{\prime}},
$$

holds for all $\mu^{\prime} \leq \mu \leq \mu^{\prime \prime}$. The choice of the interval $\left[\mu^{\prime}, \mu^{\prime \prime}\right]$ is arbitrary but must belong to the range of chemical potentials for which phase $i$ is at least metastable. The important conclusion one may draw from Eqs. (A1) and (A3) is that if the equation

$$
\omega_{i}\left(\mu_{\mathrm{x}}^{i j}\right)=\omega_{j}\left(\mu_{\mathrm{x}}^{i j}\right), \quad T=\text { constant },
$$

has a solution, there will be one and only one solution $\mu_{\mathrm{x}}^{i j}$ which is the chemical potential at which phases $i$ and $j$ coexist. Moreover, for subcritical temperatures, we anticipate $\rho_{i \mathrm{x}}$ $\neq \rho_{j \mathrm{x}}$ in general. If $\rho_{i \mathrm{x}}<\rho_{j \mathrm{x}}\left(\rho_{i \mathrm{x}}>\rho_{j \mathrm{x}}\right)$, phase $i$ will be the more (less) stable one for $\mu<\mu_{\mathrm{x}}^{i j}$ whereas phase $j$ will be the more (less) stable one for $\mu>\mu_{\mathrm{x}}^{i j}$.

${ }^{1}$ B. Jerôme, Rep. Prog. Phys. 54, 391 (1991).

${ }^{2}$ P. Sheng, Phys. Rev. Lett. 37, 1059 (1976).

${ }^{3}$ P. Sheng, Phys. Rev. A 26, 1610 (1982).

${ }^{4}$ K. Miyano, Phys. Rev. Lett. 43, 51 (1979).

${ }^{5}$ E. Grelet and H. Bock, Europhys. Lett. 73, 712 (2006).

${ }^{6}$ A. A. Sonin, The Surface Physics of Liquid Crystals (Gordon and Breach, Amsterdam, 1995).

${ }^{7}$ Y. Yi, G. Lombardo, N. Ashby, R. Barberi, J. E. Maclennan, and N. A. Clark, Phys. Rev. E 79, 041701 (2009).

${ }^{8}$ H. Hsiung, T. Rasing, and Y. R. Shen, Phys. Rev. Lett. 57, 3065 (1986).

${ }^{9}$ M. P. Valignat, N. Fraysse, and A. M. Cazabat, Langmuir 9, 3255 (1993).

${ }^{10}$ W. Chen, L. J. Martinez-Miranda, H. Hsiung, and Y. R. Shen, Phys. Rev. Lett. 62, 1860 (1989).

${ }^{11}$ R. Lucht and C. Bahr, Phys. Rev. Lett. 80, 3783 (1998).

${ }^{12}$ C. Bahr, Europhys. Lett. 88, 46001 (2009).

${ }^{13}$ X. Liu, D. W. Allender, and D. Finotello, Europhys. Lett. 59, 848 (2002).

${ }^{14}$ A. Poniewierski and T. J. Sluckin, Mol. Cryst. Liq. Cryst. 111, 373 (1983).
${ }^{15}$ T. J. Sluckin and A. Poniewierski, Phys. Rev. Lett. 55, 2907 (1985).

${ }^{16}$ A. Mauger, G. Zribi, D. L. Mills, and J. Toner, Phys. Rev. Lett. 53, 2485 (1984).

${ }^{17}$ A. K. Sen and D. E. Sullivan, Phys. Rev. A 35, 1391 (1987).

${ }^{18}$ H. Stark, J.-I. Fukuda, and H. Yokoyama, J. Phys.: Condens. Matter 16, S1911 (2004).

${ }^{19}$ P. Patrício, C.-T. Pham, and J. J. Romero-Enrique, Eur. Phys. J. E 26, 97 (2008).

${ }^{20}$ M. M. Telo da Gama, Mol. Phys. 52, 611 (1984).

${ }^{21}$ L. Onsager, Ann. N.Y. Acad. Sci. 51, 627 (1949).

${ }^{22}$ D. de las Heras, L. Mederos, and E. Velasco, Phys. Rev. E 68, 031709 (2003).

${ }^{23}$ D. de las Heras, E. Velasco, and L. Mederos, J. Chem. Phys. 120, 4949 (2004).

${ }^{24}$ M. Dijkstra, R. van Roij, and R. Evans, Phys. Rev. E 63, 051703 (2001).

${ }^{25}$ R. van Roij, M. Dijkstra, and R. Evans, Europhys. Lett. 49, 350 (2000).

${ }^{26}$ R. Zwanzig, J. Chem. Phys. 39, 1714 (1979).

${ }^{27}$ M. P. Allen, J. Chem. Phys. 112, 5447 (2000).

${ }^{28}$ M. T. Downton and M. P. Allen, Europhys. Lett. 65, 48 (2004).

${ }^{29}$ L. Bellier-Castella, D. Caprion, and J.-P. Ryckaert, J. Chem. Phys. 121, 4874 (2004).

${ }^{30}$ E. A. Müller, J. Rodriguez-Ponce, A. Qualid, J. M. Romero-Enrique, and L. F. Rull, Mol. Simul. 29, 385 (2003).

${ }^{31}$ F. Barmes and D. J. Cleaver, Phys. Rev. E 69, 061705 (2004).

${ }^{32}$ D. L. Cheung, J. Chem. Phys. 128, 194902 (2008).

${ }^{33}$ Z. Zhang, A. Chakrabarti, O. J. Mouritsen, and M. J. Zuckermann, Phys. Rev. E 53, 2461 (1996).

${ }^{34}$ S. Hess and B. Su, Z. Naturforsch. 54a, 559 (1999).

${ }^{35}$ M. Greschek, M. Melle, and M. Schoen, Soft Matter 6, 1898 (2010).

${ }^{36}$ M. Greschek and M. Schoen, Soft Matter 6, 4931 (2010).

${ }^{37}$ M. Greschek and M. Schoen, Phys. Rev. E 83, 011704 (2011).

${ }^{38} \mathrm{H}$. Steuer, S. Hess, and M. Schoen, Physica A 328, 322 (2003).

${ }^{39}$ H. Steuer, S. Hess, and M. Schoen, Phys. Rev. E 69, 031708 (2004).

${ }^{40}$ M. Schoen and S. H. L. Klapp, Reviews in Computational Chemistry (Wiley-VCH, Hoboken, 2007), Vol. 24.

${ }^{41}$ M. Schoen, O. Paris, G. Günther, D. Müter, J. Prass, and P. Fratzl, Phys. Chem. Chem. Phys. 12, 11267 (2010).

${ }^{42}$ C. G. Gray and K. E. Gubbins, Theory of Molecular Fluids (Clarendon, Oxford, 1984), Vol. 1, Chap. E 2.

${ }^{43}$ I. Pardowitz and S. Hess, Physica A 100, 540 (1980).

${ }^{44}$ W. H. Press, S. A. Teukolsky, W. T. Vetterling, and B. P. Flannery, Numerical Recipes in FORTRAN (Cambridge University Press, Cambridge, 1989), Chap. 11.1.

${ }^{45}$ A. Richter and T. Gruhn, J. Chem. Phys. 125, 064908 (2006).

${ }^{46}$ P. G. de Gennes and J. Prost, The Physics of Liquid Crystals (Oxford Science Publications, Oxford, 1995).

${ }^{47}$ R. Eppenga and D. Frenkel, Mol. Phys. 52, 1303 (1984).

${ }^{48}$ W. Maier and A. Saupe, Z. Naturforsch 14a, 882 (1959).

${ }^{49}$ W. Maier and A. Saupe, Z. Naturforsch. 15a, 287 (1960).

${ }^{50} \mathrm{G}$. Arfken, Mathematical Methods for Physicists (Academic, San Diego, 1985).

${ }^{51}$ R. Low, Eur. J. Phys. 23, 111 (2002).

${ }^{52}$ T. Gruhn and M. Schoen, Phys. Rev. E 55, 2861 (1997).

${ }^{53}$ D. J. Adams, Mol. Phys. 29, 307 (1975).

${ }^{54}$ M. P. Allen and D. J. Tildesley, Computer Simulation of Liquids (Clarendon, Oxford, 1987).

${ }^{55}$ J. H. Thurtell, M. M. Telo da Gama, and K. E. Gubbins, Mol. Phys. 54, 321 (1985).

${ }^{56}$ E. M. del Rio, M. M. Telo da Gama, and E. de Miguel, Phys. Rev. E 52, 5028 (1995).

${ }^{57}$ L. F. Rull, J. M. Romero-Enrique, and E. A. Müller, J. Phys. Chem. C 111, 15998 (2007).

${ }^{58}$ H. B. Callen, Thermodynamics (Wiley, New York, 1960). 